\title{
Nicotinamide provides neuroprotection in glaucoma by protecting against mitochondrial and metabolic dysfunction
}

\author{
James R. Tribble ${ }^{\mathrm{a}}$, Amin Otmani ${ }^{\mathrm{a}}$, Shanshan Sun ${ }^{\mathrm{b}}$, Sevannah A. Ellis ${ }^{\mathrm{c}}$, Gloria Cimaglia ${ }^{\mathrm{a}, \mathrm{b}}$, \\ Rupali Vohra ${ }^{\text {d,e }}$, Melissa Jöe ${ }^{\text {a }}$, Emma Lardner ${ }^{\text {a }}$, Abinaya P. Venkataraman ${ }^{\text {a }}$, \\ Alberto Domínguez-Vicent ${ }^{\mathrm{a}}$, Eirini Kokkali ${ }^{\mathrm{b}}$, Seungsoo Rho ${ }^{\mathrm{b}, \mathrm{f}}$, Gauti Jóhannesson ${ }^{\mathrm{g}}{ }^{\mathrm{h}}$, \\ Robert W. Burgess ${ }^{i}$, Peter G. Fuerst ${ }^{\mathrm{j}}$, Rune Brautaset ${ }^{\mathrm{a}}$, Miriam Kolko ${ }^{\mathrm{e}, \mathrm{k}}$, James E. Morgan ${ }^{\mathrm{b}, 1, \mathrm{~m}}$, \\ Jonathan G. Crowston $^{\mathrm{n}, \mathrm{o}, \mathrm{p}}$, Marcela Votruba ${ }^{\mathrm{b}, 1}$, Pete A. Williams ${ }^{\mathrm{a}, *}$
}

${ }^{\text {a }}$ Department of Clinical Neuroscience, Division of Eye and Vision, St. Erik Eye Hospital, Karolinska Institutet, Stockholm, Sweden

${ }^{\mathrm{b}}$ School of Optometry and Vision Sciences, Cardiff University, Cardiff, UK

${ }^{\mathrm{c}}$ The Florey Institute of Neuroscience and Mental Health, The University of Melbourne, Melbourne, Australia

${ }^{\mathrm{d}}$ Department of Veterinary and Animal Sciences, Pathobiological Sciences, Faculty of Health and Medical Sciences, University of Copenhagen, Denmark

${ }^{\mathrm{e}}$ Department of Drug Design and Pharmacology, Eye Translational Research Unit, Faculty of Health and Medical Sciences, University of Copenhagen, Denmark

${ }^{\mathrm{f}}$ Department of Ophthalmology, CHA Bundang Medical Center, CHA University, Seongnam, Republic of Korea

${ }^{g}$ Department of Clinical Sciences, Ophthalmology, Umeå University, Umeå, Sweden

${ }^{\mathrm{h}}$ Wallenberg Centre of Molecular Medicine, Umeå University, Umeå, Sweden

${ }^{\mathrm{i}}$ The Jackson Laboratory, Bar Harbor, ME, USA

${ }^{\mathrm{j}}$ WWAMI Medical Education Program, University of Idaho, Moscow, ID, USA

${ }^{\mathrm{k}}$ Department of Ophthalmology, Copenhagen University Hospital, Rigshospitalet-Glostrup, Glostrup, Denmark

${ }^{1}$ Cardiff Eye Unit, University Hospital Wales, Cardiff, UK

${ }^{\mathrm{m}}$ School of Medicine, Cardiff University, Cardiff, UK

${ }^{\mathrm{n}}$ Centre for Eye Research Australia, Royal Victorian Eye and Ear Hospital, Melbourne, Australia

${ }^{\circ}$ Singapore Eye Research Institute, Singapore National Eye Centre, Singapore, Singapore

${ }^{\mathrm{p}}$ Centre for Vision Research, Neuroscience and Behavioural Disorders, Duke-NUS, Singapore, Singapore

\section{A R T I C L E I N F O}

\section{Keywords:}

Glaucoma

Retina

Retinal ganglion cell

Nicotinamide

Metabolism

Metabolomics

Mitochondria

\begin{abstract}
A B S T R A C T
Nicotinamide adenine dinucleotide (NAD) is a REDOX cofactor and metabolite essential for neuronal survival. Glaucoma is a common neurodegenerative disease in which neuronal levels of NAD decline. We assess the effects of nicotinamide (a precursor to NAD) on retinal ganglion cells (the affected neuron in glaucoma) in normal physiological conditions and across a range of glaucoma relevant insults including mitochondrial stress and axon degenerative insults. We demonstrate retinal ganglion cell somal, axonal, and dendritic neuroprotection by nicotinamide in rodent models which represent isolated ocular hypertensive, axon degenerative, and mitochondrial degenerative insults. We performed metabolomics enriched for small molecular weight metabolites for the retina, optic nerve, and superior colliculus which demonstrates that ocular hypertension induces widespread metabolic disruption, including consistent changes to $\alpha$-ketoglutaric acid, creatine/creatinine, homocysteine, and glycerophosphocholine. This metabolic disruption is prevented by nicotinamide. Nicotinamide provides further neuroprotective effects by increasing oxidative phosphorylation, buffering and preventing metabolic stress, and increasing mitochondrial size and motility whilst simultaneously dampening action potential firing frequency. These data support continued determination of the utility of long-term nicotinamide treatment as a neuroprotective therapy for human glaucoma.
\end{abstract}

\footnotetext{
* Corresponding author.

E-mail addresses: james.tribble@ki.se (J.R. Tribble), amin.otmani@ki.se (A. Otmani), SunS9@cardiff.ac.uk (S. Sun), ellissa@student.unimelb.edu.au (S.A. Ellis),

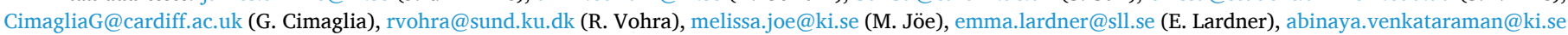

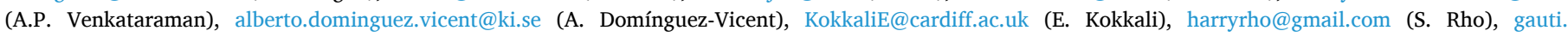

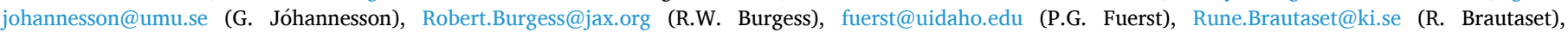

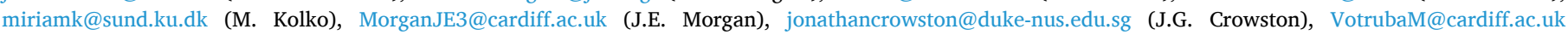
(M. Votruba), pete.williams@ki.se (P.A. Williams).
} 


\section{Background}

Glaucoma is one of the most common neurodegenerative diseases, and the leading cause of irreversible blindness, affecting $\sim 80$ million people worldwide [1]. Age, genetics, and elevated intraocular pressure (IOP) are major risk factors, but to date, the only clinically available therapies target the reduction of IOP. For the many patients which are refractory to IOP lowering treatments, surgical interventions are commonly used in order to limit the progressive neurodegeneration and visual impairment [2]. No therapy currently targets the neuronal population that degenerates in glaucoma. Ultimately, $42 \%$ of treated glaucoma patients progress to blindness in at least one eye [3]. With an increasingly aged population, glaucoma will continue to be a significant health and economic burden and thus neuroprotective strategies targeting the retina and optic nerve are of great therapeutic need.

Retinal ganglion cells, the output neuron of the retina whose axons converge to form the optic nerve, display a progressive, compartmentalized neurodegeneration leading to the characteristic visual dysfunction seen in glaucoma. We have previously demonstrated mitochondrial abnormalities occurring prior to neurodegeneration in glaucoma (in glaucoma patients and animal models [4,5]). The latter study, utilizing the DBA/2J mouse model of glaucoma, has identified that nicotinamide adenine dinucleotide (NAD; an essential REDOX cofactor and metabolite) declines in the retina in an age-dependent manner and renders retinal ganglion cells susceptible to IOP-related stress, driving glaucomatous neurodegeneration. The prevention of NAD decline by dietary supplementation with nicotinamide (NAM; the amide form of vitamin $\mathrm{B}_{3}$, an NAD precursor via the NAD-salvage pathway in neurons) or by intravitreal viral gene-therapy overexpressing Nmnat1 (a terminal enzyme for NAD production) was robustly protective against retinal ganglion cell neurodegeneration and prevented a number of early gene expression changes in DBA/2J retinal ganglion cells [5]. Glaucoma patients have been demonstrated to have systemically low levels of NAM in sera [6] which supports a hypothesis in which pathogenically low NAD leads to glaucoma susceptibility. These findings have driven interest in nicotinamide as a treatment for glaucoma, including the planning and initiation of a number of clinical trials, e.g. NCT03797469 which is ongoing and Hui et al. (2020) which is now completed [7]. In this trial, as part of a multi-national collaborative team, we have demonstrated significant visual recovery in glaucoma patients over a 6 month treatment period (cross-over design; step up $1.5 \mathrm{~g} / \mathrm{d}$ to $3.0 \mathrm{~g} / \mathrm{d}$ nicotinamide [7]). Nicotinamide's excellent long clinical history, combined with good safety profile, tolerance at high doses, and affordability has facilitated rapid translation into initial small scale clinical trials [8,9]. However, translation to larger scale trials, the evaluation of earlier intervention protocols, and/or longer-term clinical outcomes requires a deeper understanding of the effects of nicotinamide on retinal ganglion cells under both neurodegenerative conditions and normal physiological states. This is of critical importance since disease progression is often heterogeneous (i.e. a mix of healthy, degenerating, and dead cells) and if prophylactic treatment is to be considered (which is ideal since visual deficits present once the degenerative processes have already been initiated [10]).

We bridge the gap between the bench and bedside by demonstrating further evidence of neuroprotection through nicotinamide supplementation across a number of glaucoma-related retinal ganglion cell targeted insults, using a number of retinal ganglion cell specific tools. We identify early metabolic changes in the retina in a rat model of ocular hypertensive glaucoma (elevated IOP), which are prevented by nicotinamide supplementation. Importantly, chronic dietary nicotinamide supplementation causes limited metabolic variation in non-diseased visual system tissues supporting potential prophylactic treatments. Nicotinamide administration also buffers retinal ganglion cell metabolism, increases oxidative phosphorylation, and positively alters mitochondrial morphology and dynamics in retinal ganglion cells at doses which are neuroprotective. These data help support nicotinamide's use in the clinic, and support the initiation of clinical trials to determine the effect of nicotinamide on longer-term disease progression.

\section{Materials and methods}

\subsection{Animal strain and husbandry}

All breeding and experimental procedures were undertaken in accordance with the Association for Research for Vision and Ophthalmology Statement for the Use of Animals in Ophthalmic and Research. Individual study protocols were approved by Stockholm's Committee for Ethical Animal Research (10389-2018). Animals were housed and fed in

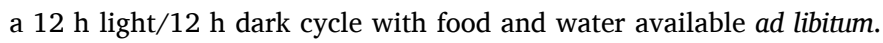
Adult, male Brown Norway rats (BN, aged 12-20 weeks, weighing 300-400 g, SCANBUR) were utilized for inducing ocular hypertension (detailed below). C57BL/6J and MitoV (strain details and generation detailed below) mouse strains were bred and utilized at 12-20 weeks of age. For rats, Nicotinamide (NAM, PanReac AppliChem) was dissolved in drinking water $(200 \mathrm{mg} / \mathrm{kg} / \mathrm{d}$, based on average consumption). Higher doses were achieved by combining treated drinking water and a custom diet (3130 ppm NAM to achieve $400 \mathrm{mg} / \mathrm{kg} / \mathrm{d}$, or $9380 \mathrm{ppm}$ NAM to achieve $800 \mathrm{mg} / \mathrm{kg} / \mathrm{d}$ based on a pre-determined $\mathrm{g} / \mathrm{d}$ consumption of chow, base RM3 P diet, SDS). For mice, NAM was dissolved in drinking water $(500 \mathrm{mg} / \mathrm{kg} / \mathrm{d}$ based on average consumption). Water was protected from light and changed every 3-5 days and chow was maintained as necessary; both were provided in sufficient quantity to be available ad libitum.

\subsection{Induction and monitoring of rat ocular hypertension}

Rat ocular hypertension was induced using a paramagnetic bead model as previously described [11]. Rats were anesthetized with an intraperitoneal injection of ketamine $(37.5 \mathrm{mg} / \mathrm{kg})$ and medetomidine hydrochloride $(1.25 \mathrm{mg} / \mathrm{kg}$ ). Microbeads (Dynabead Epoxy M - 450, Thermo Fisher) were prepared in $1 \mathrm{x}$ Hank's balanced salt solution (HBSS $-\mathrm{CaCl}_{2}-\mathrm{MgCl}_{2}$-phenol red, Gibco) and 6-8 $\mu \mathrm{l}$ of bead solution was injected into the anterior chamber. Beads were directed with a magnet to block the iridocorneal angle. Rats received either bilateral injections (OHT) or remained bilateral un-operated (naïve), normotensive controls (NT). For rats pre-treated with NAM, NAM was given 1 week prior OHT induction (at either 200, 400, or $800 \mathrm{mg} / \mathrm{kg} / \mathrm{d}$ ) and maintained throughout the duration of the experiment. Intraocular pressure (IOP) was measured using a rebound tonometer (Tonolab, Icare). Rats were habituated to tonometry in the week prior to induction. Baseline IOP was recorded the morning before surgery and every 2-4 days afterwards. Rats were awake and unrestrained for tonometry. IOP recordings were always performed between 9 and 10 am to avoid the effects of the circadian rhythm on IOP.

\subsection{OCT imaging}

Optical coherence tomography (OCT) was performed at 14 days post IOP in BN rats, prior to euthanasia ( $\mathrm{n}=9$ eyes NT, 7 OHT, 8 OHT treated with $200 \mathrm{mg} / \mathrm{kg} / \mathrm{d}$ NAM (OHT-NAM)). Pupils were dilated with topical Phenylephrine Hydrochloride (2.5\% w/v) eye drops before anesthesia was induced with an intraperitoneal injection of ketamine and medetomidine hydrochloride as above. OCT imaging was performed using a Phoenix Micron IV (Phoenix) with image-guided OCT attachment. Bscans centered on the $\mathrm{ONH}$ were captured in the horizontal and vertical plane. Image analysis was performed in MATLAB (MATrix LABoratory). First the retinal pigment epithelium and internal limiting membrane were delineated manually. In the presence of cupping, the cup depth was measured automatically as the vertical distance between the cup base and midpoint of the neuroretinal peaks. This measurement was used to determine loss of neuroretinal rim. 


\subsection{Retina axotomy explant model}

A retinal axotomy model was established with modification form previously described protocols [12,13]. Mice were euthanized by cervical dislocation and eyes immediately enucleated. Retinas were dissected free in cold HBSS and flat mounted on cell culture inserts (Millicell $0.4 \mu \mathrm{m}$ pore; Merck) ganglion cell layer up. Retinas were maintained in culture $\left(37{ }^{\circ} \mathrm{C}, 5 \% \mathrm{CO}_{2}\right)$ fed by Neurobasal-A media supplemented with $2 \mathrm{mM}$ L-glutamate (GlutaMAX), $2 \% \mathrm{~B} 27,1 \% \mathrm{~N} 2$, and $1 \%$ penicillin/streptomycin (all Gibco) in 6-well culture plates. Half the media volume was changed after 2 days. For NAM treated retinas, NAM was dissolved in the culture media to a concentration of $100 \mathrm{mM}$ or 500 $\mathrm{mM}$. Retinas were removed from culture for further processing after 12 h, 1 day, 3 days or 5 days ex vivo (DEV).

\subsection{Intravitreal rotenone model}

Rotenone toxicity induced degeneration was induced in the retina following established protocols $[14,15]$. Mice were anesthetized with an intraperitoneal injection of ketamine $(37.5 \mathrm{mg} / \mathrm{kg})$ and medetomidine hydrochloride $(1.25 \mathrm{mg} / \mathrm{kg})$. Bilateral intravitreal injections were performed using a $33 \mathrm{G}$ tri-beveled needle on a $10 \mu \mathrm{l}$ glass syringe (WPI). One $\mu \mathrm{l}$ of a $10 \mathrm{mM}$ rotenone (MP Biochemicals) solution dissolved in DMSO (PanReac AppliChem) or DMSO only (vehicle only control) was injected into the vitreous and the needle maintained in place for $30 \mathrm{~s}$ for distribution. For NAM pre-treated retinas, NAM $(500 \mathrm{mg} / \mathrm{kg} / \mathrm{d})$ was administered in drinking water 1 week prior to rotenone injection. Mice were euthanized $24 \mathrm{~h}$ after injection by cervical dislocation and eyes immediately enucleated for further processing.

\subsection{Cryo-sectioning}

Rats were heavily anesthetized by intraperitoneal injection of pentobarbital $(75 \mathrm{mg} / \mathrm{kg}$ ) and euthanized by cervical dislocation. Mice were euthanized by cervical dislocation. Eyes for cryo-sectioning were enucleated and fixed by immersion in $4.7 \%$ PFA for $24 \mathrm{~h}$. The tissue was cryo-protected by immersion in 30\% sucrose for $24 \mathrm{~h}$ before freezing in optimal cutting temperature medium (Sakura) on dry ice. Blocks were maintained at $-80{ }^{\circ} \mathrm{C}$ until use. $20 \mu \mathrm{m}$ cryo-sections were cut (anterior to dorsal plane) using a cryostat (Cryostar NX70, Thermo Scientific) and stored at $-20^{\circ} \mathrm{C}$.

\subsection{Immunofluorescent labelling}

Immunofluorescent labelling followed a standard protocol for cryosections and flat mount retina with the following exceptions. Cryosections were air dried for $15 \mathrm{~min}$ and rehydrated in $1 \mathrm{M}$ PBS for 5 min before following the protocol. Antibodies are detailed in Supplementary Table 1. For the standard protocol, tissue was isolated using a hydrophobic barrier pen (VWR), permeabilized with 0.5\% Triton X-100 (VWR) in $1 \mathrm{M}$ PBS for $30 \mathrm{~min}$, blocked in $5 \%$ bovine serum albumin (Fisher Scientific) in $1 \mathrm{M}$ PBS for $30 \mathrm{~min}$, and primary antibody applied and maintained overnight at $4{ }^{\circ} \mathrm{C} .5 \times 5$ min washes in 1 M PBS were performed before applying secondary antibodies and maintaining at room temperature for $2 \mathrm{~h}$. Tissue was washed as before and DAPI nuclear stain $(1 \mu \mathrm{g} / \mathrm{ml}$ in $1 \mathrm{M}$ PBS$)$ was applied for $10 \mathrm{~min}$. Tissue was then washed once in PBS before mounted using Fluoromount-G and glass coverslips (Invitrogen). Slides were sealed with nail-varnish.

\subsection{Analysis of RGC somal death and degeneration}

RGC loss and nuclear shrinkage was assessed through RBPMS and DAPI labelling of flat mounts. Images were acquired on a Zeiss Axioskop 2 plus epifluorescence microscope (Karl Zeiss). Six images per retina (40X magnification, $0.25 \mu \mathrm{m} / \mathrm{pixel}$ ) were taken equidistant at $0,2,4,6$, 8 , and 10 o'clock about a superior to inferior line through the optic nerve head $\left(\sim 1000 \mu \mathrm{m}\right.$ eccentricity). Images were cropped to $150 \mu \mathrm{m}^{2}$ and RBPMS + cells and DAPI nuclei counted using the cell counter plugin for Fiji ([16]; only round nuclei were counted, thus excluding vascular endothelium); counts were expressed as a density per 0.01 $\mathrm{mm}^{2}$, averaged across the 6 images. For measurement of nuclear diameter, 30 nuclei (from RBPMS + cells; or as many surviving cells as were able to be measured) per cropped image were measured using the line tool, giving an average diameter; this was averaged across the 6 images to produce a final average diameter per retina. These analyses were performed on rat retina $(n=10$ eyes from NT, 10 eyes from OHT, 9 eyes from OHT-NAM [200 mg/kg/d], 12 eyes from OHT-NAM [400 $\mathrm{mg} / \mathrm{kg} / \mathrm{d}$ ], 12 eyes from OHT-NAM [800 mg/kg/d]), C57BL/6J mouse retina from retinal explants $(\mathrm{n}=6$ eyes for all groups: 0 days ex vivo (DEV; control), 0.5 DEV, 1 DEV, 3 DEV, 5 DEV, 3 DEV + NAM [100 mM], $3 \mathrm{DEV}+\mathrm{NAM}[500 \mathrm{mM}]), \mathrm{C} 57 \mathrm{BL} / 6 \mathrm{~J}$ mouse retina from intravitreal rotenone injection ( $\mathrm{n}=8$ eyes from DMSO control, 8 eyes from rotenone injected, 8 eyes from rotenone + NAM $[500 \mathrm{mg} / \mathrm{kg} / \mathrm{d}])$.

\subsection{Analysis of RGC axonal degeneration}

Axon integrity in the nerve fiber layer (NFL) of retina following axotomy explant was assessed relative to control $(n=6$ eyes for all groups: 0 days ex vivo (DEV; control), 3 DEV, 3 DEV + NAM [100 mM], 3 DEV + NAM [500 mM]). Images of $\beta$-tubulin labelled axons adjacent to the optic nerve head were acquired on a Zeiss Axioskop 2 plus epifluorescence microscope, cropped to $250 \times 50 \mu \mathrm{m}$ size and the size and number of varicosities measured. Images were converted to 8 bit, thresholded (200-255) and binarised. Varicosities were analyzed using the ImageJ particle analyzer (Fiji; $1-10 \mu \mathrm{m}$ size filter to remove single pixel and long, thin axon segments [17]). The number of particles $/ \mathrm{mm}^{2}$ and the average size of particles $\left(\mu \mathrm{m}^{2}\right)$ were taken as varicosity density and size.

\subsection{Analysis of RGC dendritic degeneration}

Dendritic degeneration was assessed in axotomy explant retina through DiOlistic labelling of individual RGC dendritic arbors (using a Helios gene gun system, Biorad, as previously described [18]). Bullets were prepared at a ratio of $2 \mathrm{mg}$ DiI, $4 \mathrm{mg}$ of DiO, and $80 \mathrm{mg}$ Tungsten (1.7 $\mu \mathrm{m}$ diameter) to $30.5 \mathrm{~cm}$ of Tezfel tubing (Biorad), by dissolving the dyes in $400 \mu \mathrm{l}$ of methylene chloride and applying over the tungsten to air dry. The coted tungsten was collected and transferred into the tubing, and distributed along the length of the tubing by vortexing. Individual bullets were cut to $1.2 \mathrm{~cm} \mathrm{C57BL/6J}$ mouse retinas were subject to axotomy explant ( $\mathrm{n}=10$ eyes for 0 days ex vivo (DEV; control), 12 eyes for all 3 DEV groups: 3 DEV, 3 DEV + NAM [100 mM], 3 DEV + NAM [500 mM]) and labelled ballistically. For $0 \mathrm{DEV}$, retinas were dissected in HBSS and immediately labelled, for 3 DEV groups retinas were removed from culture and labelled. For labelling, retinas were flat mounted on to glass slides, all liquid was removed, a culture insert was inverted over the retina to act as a filter to large particle clumps, and the contents of a single bullet discharged at a gene gun pressure of $120 \mathrm{psi}$. Tissue was transferred to a culture dish containing Neurobasal-A media (supplemented as in the retina axotomy explant model above) and maintained at $37{ }^{\circ} \mathrm{C}, 5 \% \mathrm{CO}_{2}$ for $30 \mathrm{~min}$. Retinas were then fixed in $4 \%$ PFA for $1 \mathrm{~h}$, washed in $1 \mathrm{M}$ PBS, nuclei labelled with Hoechst (Invitrogen) for $15 \mathrm{~min}$, washed again and mounted with FluorSave reagent (Merck). After drying, coverslips were sealed. Images of individual RGC dendritic arbors were acquired on a Leica SP8 confocal microscope (Leica Systems; 20X magnification, $0.45 \mu \mathrm{m} /$ pixel, $1 \mu \mathrm{m} z$-thickness). Whole dendritic arbors were reconstructed using Imaris (version 9.3.1, Bitplane) where individual RGC dendritic fields were manually selected as areas of interest (used to calculate dendritic field areas) and dendrites automatically traced using the filaments tool. Total dendritic length was calculated and Sholl analysis performed (dendritic intersections per binned distance from the soma center, $10 \mu \mathrm{m}$ steps) and area under the 
curve (AUC) of the Sholl curve was calculated. Dendritic health and integrity was evaluated by quantifying dendrite varicosities. Varicosities were identified automatically by the spot tool and a density per cell calculated by normalizing to total dendritic length and dendritic field area.

\subsection{Mitochondrial RNA to nuclear RNA ratio}

The ratio of RNA from genes encoded in the mitochondrial (mt) and nuclear (nu) genome can differential expression of mitochondrial genes or loss/gain of mitochondrial numbers [19]. Six C57BL/6J mouse retina following 3 days ex vivo of the retina axotomy explant model, and six 0 DEV controls were homogenized into $400 \mu$ l buffer RLT (Qiagen) with $1 \% \beta$-mercaptoethanol (Fisher Scientific) using a QIAshredder kit (Qiagen) according to the manufacturer's instructions. RNA was extracted using RNeasy Mini Kits (Qiagen) according to the manufacturer's instructions. RNA was extracted into nuclease-free water, and RNA concentration was measured in a $1 \mu$ sample diluted 1:200 in nuclease-free water in a spectrophotometer (BioPhotometer, Eppendorf). cDNA was synthesized using $1 \mu \mathrm{g}$ of input RNA with an iScript ${ }^{\mathrm{TM}}$ cDNA Synthesis Kit and MyIQ thermocycler (both Bio-Rad) and stored at $-20{ }^{\circ} \mathrm{C}$ overnight. RT-qPCR was performed using $1 \mu \mathrm{g}$ of input cDNA, $7.5 \mu \mathrm{l}$ of SsoAdvanced Universal SYBR Green Supermix and $1 \mu \mathrm{l}$ of the following DNA templates (Prime PCR Assay, Bio-Rad): $m t-C o 2$ (mitochondrial; mus musculus), Rps18 (nuclear; mus musculus), and Tbp (housekeeping; mus musculus). A MyIQ thermocycler was used with a 3 min activation and denaturation step at $95{ }^{\circ} \mathrm{C}$, followed by an amplification stage comprising 50 cycles of a $15 \mathrm{~s}$ denaturation at $95{ }^{\circ} \mathrm{C}$ and 1 min annealing and plate read at $60{ }^{\circ} \mathrm{C}$. Analysis was performed according to the $\Delta \Delta \mathrm{CT}$ method outlined by Quiros et al. [19] giving a mtRNA:nuRNA ratio. $\Delta \Delta$ CTs were compared by student's $t$-test.

\subsection{Metabolomics}

BN rats were induced with OHT for 3 days or remained as NT controls (as described above). Rats were either pretreated with NAM (800 $\mathrm{mg} / \mathrm{kg} / \mathrm{d}$ ) for 7 days (10 days total exposure) or were untreated ( $\mathrm{n}=8$ eyes NT, $\mathrm{n}=8$ eyes OHT, $\mathrm{n}=8$ eyes NT-NAM, $\mathrm{n}=8$ eyes OHT-NAM). Rats were euthanized by pentobarbital injection and cervical dislocation (as described above). Retinas were immediately dissected on ice cold HBSS (optic nerve head was not included), wiped dry and weighed before snap freezing on dry ice. A second investigator removed the brain and attached optic nerves immediately after death. Optic nerves were separated at the chiasm and weighed before freezing as for retinas. The superior colliculus (SC) was isolated and separated into left and right hemispheres before weighing and freezing as for the retina. Tissue was stored on dry ice and shipped to the Swedish Metabolomics Centre for sample processing. $200 \mu \mathrm{l}$ of $90: 10 \mathrm{MeOH}: \mathrm{H}_{2} \mathrm{O}$ was added to each frozen sample on dry ice, acid washed glass-beads (425-600 $\mu \mathrm{m}$; Sigma Aldrich) were added to constitute $50 \% \mathrm{v} / \mathrm{v}$ of the $\mathrm{MeOH}: \mathrm{H}_{2} \mathrm{O}$ solution and tissues were disrupted by shaking at $30 \mathrm{~Hz}$ for $3 \mathrm{~min}$, followed by an additional shaking step after the addition of $100 \mu \mathrm{l}$ of $\mathrm{H}_{2} \mathrm{O}$. Samples were then centrifuged (14,000 RPM, $10 \mathrm{~min}, 4^{\circ} \mathrm{C}$ ) and $100 \mu \mathrm{l}$ of the supernatant was transferred to HPLC vials and immediately analyzed. $2 \mu \mathrm{l}$ was injected into an Agilent 1290 UPLC-system connected to an Agilent 6550 Q-TOF mass spectrometer with an Agilent Jet Stream electrospray ionization (ESI) source. Data was collected in positive and negative ionization mode. The HPLC column used was an HILIC (iHILIC-Fusion (+), $100 \times 2.1 \mathrm{~mm}, 3.5 \mu \mathrm{M}, 100 \AA$, Hilicon AB). HILIC elution solvents used were A) $50 \mathrm{mM}$ ammonium formate in $\mathrm{H}_{2} \mathrm{O}$ and B) 90:10 Acetonitrile: [50 mM ammonium formate in $\mathrm{H}_{2} \mathrm{O}$ ]. Chromatographic separation at a flow rate $0.4 \mathrm{~mL} / \mathrm{min}$ was achieved using the following linear gradient: $\min 0=90 \% \mathrm{~B}, \min 4=85 \% \mathrm{~B}, \min 5=70 \% \mathrm{~B}, \min 7=55 \%$ $B, \min 10=20 \% B, \min 10.01=90 \% B, \min 15=90 \%$ B. Seventy three low molecular weight metabolites that could be verified with standards were detected. Metabolites were quantified as area under the curve of the mass spectrometry peak and normalized to an internal standard for negative and positive runs, then normalized to tissue weight. Data were analyzed using MetaboAnalyst (version 4.0 [20,21]). Groups were compared by two-sample $t$-tests with an adjusted $P$ value (false discovery rate, FDR) cutoff of 0.05 deemed significant. Dendograms were created using hierarchical clustering (HC) in R (1-cor, Spearman's rho). Heatmaps were created in $\mathrm{R}$ and using Morpheus (https://software.br oadinstitute.org/morpheus). Principle component analysis was calculated using Pareto scaling to reduce the effect of magnitude [22], without excluding this dimension entirely. Pathways analysis was performed in MetaboAnalyst using the Rattus norvegicus KEGG library. Circos plots were created in $\mathrm{R}$ using the circlize package [23], using Spearman's rank correlations with a $P$ value adjusted for multiple comparisons.

\subsection{Luminometry-based NAD assays}

To measure NAD concentration in retina and optic nerves following 14 days of OHT, BN rats were anesthetized by intraperitoneal injection of pentobarbital $(75 \mathrm{mg} / \mathrm{kg}$ ), and euthanized by cervical dislocation ( $\mathrm{n}$ $=6$ eyes and optic nerves from NT, 6 eyes and optic nerves from OHT). Whole eyes were removed and retina dissected in HBSS as described above. The brain was removed with attached optic nerves. Optic nerves were cut at the chiasm and $4 \mathrm{~mm}$ from the end proximal to the eye was measured and collected and stored at $4{ }^{\circ} \mathrm{C}$ in HBSS until further processing. Retinas were transferred to $500 \mu \mathrm{l}$ of dispase (5000 U, Corning) and put on a heating block (Thermomixer C, Eppendorf) at $37^{\circ} \mathrm{C}, 350$ rpm for $30 \mathrm{~min}$ before dissociation by gentle trituration. Cell concentration was determined by cell counting on a hemocytometer (C-Chip, NanoEntek) and each sample was diluted to a concentration of 2 million cells $/ \mathrm{ml}$. Retina and optic nerve samples were then homogenized for 20 $\mathrm{s}, 30,000 \mathrm{~min}^{-1}$ (VDI 12, VWR). NAD concentration was performed using a bioluminescent assay (NAD/NADH Glo-TM, Promega). Kit reagents were prepared according to the manufacturer's instructions. NAD-standards were prepared in HBSS from a $2 \mathrm{mM}$ NAD stock (no. N8285, Sigma-Aldrich). Either $50 \mu 1$ of sample (100,000 cells/well for retina) or NAD standard were combined with $50 \mu \mathrm{l}$ of reagent in a 96well plate (Nunc ${ }^{\mathrm{TM}}$ F96 MicroWell ${ }^{\mathrm{TM}}$ White Polystyrene plate, ThermoFisher Scientific). Luminescence was recorded at approx. $1 \mathrm{~h}$ from initial mixing (plate shake in machine) to give sufficient time for the reaction to occur and remain stable. Luminescent intensity was converted to NAD concentration using the NAD standard curve. In order to assess how quickly nicotinamide could be utilized by cells to increase NAD concentration NAD assays were performed on treated retina, optic nerve, and brain cortex. C57BL/6J mice ( $\mathrm{n}=4$ mice) were euthanized by cervical dislocation and eyes and optic nerve dissected as above with the exceptions that retinas from left and right eyes were pooled to form a single sample (giving a concentration after processing of 1.4 million cells $/ \mathrm{ml}$ ) and where optic nerves were cut to $2 \mathrm{~mm}$. Whole cortex was removed, separated by hemisphere, transferred to $800 \mu \mathrm{l}$ of dispase and processed as for the retina above to achieve a concentration of 2 million cells/ml. From each sample (retina, optic nerve and cortex) 4 aliquots of cell homogenate were taken for incubation with NAM $(0 \mathrm{mM}, 0.1 \mathrm{mM}$, $10 \mathrm{mM}$ ) for either $0,2,4$, or $6 \mathrm{~h}$. NAM was added to the correct concentration to the samples so that all incubation times would complete at the same time point. The bioluminescent assay was then conducted as above.

\subsection{Generation and characterization of MitoV mouse}

MitoV mice were an independent founder (substrain 1819) in the generation of transgenic mice described by Misgeld et al. and as detailed by Burgess and Fuerst $[24,25]$. YFP is expressed under a rat Eno2 promoter (neuron specific) and localized to mitochondria via a Cox8a gene-targeting signal fused to the YFP N-terminus. Mice are on a C57BL/6J background. We selected this line based on specificity of inner 
retinal expression (designated MitoV for visual tissue). Four week old mice were euthanized by cervical dislocation, eyes were enucleated and fixed in PFA for $1 \mathrm{~h}$, before performing immunofluorescent labelling as described above with anti-GFP and anti-RBPMS antibodies. Brains were fixed in PFA and processed for whole brain cryo-sectioning as described above.

\subsection{Super-resolution fluorescent imaging of mitochondria and morphological analysis}

Super-resolution fluorescent images of mitochondria were captured using Airyscan imaging on a Zeiss LSM800-Airy (63X, 1.3X optical zoom, $66.76 \times 66.76 \mu \mathrm{m}$ images, $35 \mathrm{~nm} /$ pixel, z-stacks with $15 \mathrm{~nm}$ slice thickness). For MitoV mouse tissue, images were acquired of Alexa Fluor 488 conjugated secondary antibodies targeting anti-GFP primary antibody to limit loss of signal from potential YFP bleaching. To allow for practical acquisition times, only the mitochondrial channel was imaged; DAPI nuclear stain and RBPMS labelling in the same image window were captured as snapshots only for reference purposes. Secondary antibody only controls were used for all experiments to set suitable imaging parameters. Retina axotomy explant was performed on MitoV retinas, with 4 retinas maintained for $0 \mathrm{DEV}$ (control), 4 retinas for $0.5 \mathrm{DEV}$, and 4 retinas for $0.5 \mathrm{DEV}$ with NAM supplemented media $(500 \mathrm{mM})$. Retinas were imaged as flat mounts, with 4 images collected at $1000 \mu \mathrm{m}$ eccentricity from the optic nerve head superiorly, nasally, inferiorly, and temporally. Images were captured as z-stacks from the NFL to the lower boundary of the GCL. The intravitreal rotenone injection model was performed with 9 DMSO (vehicle only) injected retinas, 4 Rotenone (10 $\mathrm{mM}$ ) injected retinas and 7 Rotenone injected retinas where animals were pre-treated with NAM $(500 \mathrm{mg} / \mathrm{kg} / \mathrm{d})$ for 1 week prior to injection and maintained for the duration of the experiment. Eyes were enucleated $24 \mathrm{~h}$ after injection, fixed in PFA and processed for cryo-sectioning as described above. $20 \mathrm{~m}$ cryosections were cut and immunofluorescent labelling performed using Alexa Fluor 488 conjugated secondary antibodies targeting anti-GFP primary antibody. Airyscan images were acquired of central retina $\sim 500 \mu \mathrm{m}$ later to the optic nerve head, encompassing the NFL, GCL and inner plexiform layer (IPL). The IPL was cropped for analysis. In rat tissue, 5 NT eyes (from 3 rats), 6 OHT eyes (from 4 rats) and 4 OHT-NAM (from 4 rats, pre-treatment with $800 \mathrm{mg}$ / $\mathrm{kg} / \mathrm{d}$ NAM) were collected 14 days after OHT induction and processed for cryo-sectioning as described above. Alexa Fluor 568 conjugated secondary antibodies targeting anti-TOMM20 primary antibodies was imaged. Image capture and analysis followed the same protocol as for rotenone injected eyes. IPL and GCL/NFL crops were analyzed separately. In all three models, mitochondria were reconstructed in 3D using Imaris software (version 9.3.1). Volume reconstructions were performed using the surfaces tool and individual volume and surface area were calculated. Volumes $<125$ voxels were filtered and removed from subsequent analysis to reduce noise.

\subsection{Live-imaging of mitochondria}

RGC cultures were established according as previously described ([26], modified from [27]). Six to seven day old C57BL/6J pups were euthanized and retinas dissected $(n=16-18$ per panning procedure, $3 \mathrm{X}$ repeats). Retinas were dissociated in DPBS (Thermofisher, UK) by forceps within $35 \mathrm{~min}$ at room temperature under $4 \mathrm{X}$ dissecting microscope followed by gentle trituration. RGCs were purified using two-step immune-panning on two Lectin coated negative panning plates (Bandeiraea simplicifolia, BSL-1; Vector Laboratories Ltd.) and a Thy1.2 antibody coated positive plate (Serotec MCA02R, Bio-Rad), over $45 \mathrm{~min}$ at room temperature $\left(25^{\circ} \mathrm{C}\right)$. RGCs were seeded at a density of 50,000 cells per well (24 well plate; Nunc, Thermofisher) on Poly-D-lysine and laminin coated glass coverslips (Sigma-Aldrich, R\&D Systems, and Academy Science Limited, respectively). RGCs were cultured in serum-free growth medium $\left(37^{\circ} \mathrm{C}, 10 \% \mathrm{CO}_{2}\right)$ for 10 wells as previously described and treated with NAM dissolved in the media at concentrations of $0,50,100,500$, and $1000 \mu \mathrm{M}$ at day 7 post seeding. At eight days post seeding, mitochondria were stained with MitoTracker Red CMXRos (Molecular Probes, Invitrogen) for $30 \mathrm{~min}$ at $37^{\circ} \mathrm{C}$. Using a Zeiss LSM 880 confocal microscope (63X oil immersion lens, $0.132 \mu \mathrm{m} / \mathrm{px}$ ) time-lapse imaging was performed in order to record mitochondrial movement. Images were captured every $5 \mathrm{~s}$ for $10 \mathrm{~min}$. Mitochondria were classified as either stationary or moving over the $10 \mathrm{~min}$ recording based on a total $5 \mu \mathrm{m}$ distance threshold. Mitochondrial length (long axis), and number of mitochondria (total, motile, stationary) in the entire length of neurites in each group was measured in the first frame (Fiji) and Kymographs of mitochondrial movements were generated using the KymoAnlyzer plugin [28] for Fiji. Mitochondrial velocity was calculated as distance travelled on the kymograph $x$ axis divided by length of the $y$ axis (duration of movement).

\subsection{Extracellular acidification rate and oxygen consumption rate}

RGCs were purified from mice according to the methods previously described by Skytt et al. and Vohra et al. [29,30]. Culture media was a combination of Neurobasal A medium and DMEM, supplemented with glucose to reach a final concentration of $6 \mathrm{mM}$, and with penicillin/streptomycine, sato, T3, B27, NAC, Trace elements B, biotin, CNTF, BDNF, forskolin, FGFB, and insulin as described in the references above. RGCs were cultured in a Seahorse 96-well cell culture microplate at a density corresponding to 450,000 cells $/ \mathrm{cm}^{2}$. Glycolytic and mitochondrial respiratory function was measured using real-time assessment of, respectively, the extracellular acidification rate (ECAR) and oxygen consumption rate (OCR) with the Seahorse XFe96 Extracellular Flux Analyzer (Seahorse Biosciences-Agilent Technologies, USA) after one week. On the day prior to the assay, cells were pre-incubated $24 \mathrm{~h}$ before the analysis under the different experimental conditions: Control $(0 \mu \mathrm{M}$ nicotinamide (NAM)), $50 \mu \mathrm{M}$ NAM, $500 \mu \mathrm{M}$ NAM and $1000 \mu \mathrm{M}$ NAM in culture media as above (no pyruvate, no glutamine) Prior to the assay, culture media was changed to $\mathrm{CO}_{2}$ calibrated Seahorse XF DMEM (unbuffered, adjusted to $\mathrm{pH} 7.4$, supplemented with glucose to reach a final concentration of $6 \mathrm{mM}$ ) and the cells were equilibrated for $15 \mathrm{~min}$ at $37{ }^{\circ} \mathrm{C}$ in a $\mathrm{CO}_{2}$-free incubator. Time of calibration inside the seahorse instrument was set for further $15 \mathrm{~min}$. Each OCR measurement cycle consisted of 3-min mix and 3-min measurement of the oxygen level, as well as ECR. The analysis of glycolytic and mitochondrial function was initiated by three baseline OCR measurement cycles. The $\mathrm{pH}$ of the reagents used to test mitochondrial function was adjusted to 7.4. The OCR and ECR were recorded and calculated by the Seahorse XFe96 software, Wave (Seahorse Biosciences-Agilent Technologies, USA). To normalize the data after the Seahorse analysis, the protein content for each well was measured using a BCA assay with BSA as standard. Measurements were acquired for 3-5 different cell batches per condition.

\subsection{Whole-cell patch-clamp electrophysiology}

C57BL/6J mice (12 weeks) were anesthetized with $2 \%$ isoflurane and cervically dislocated. Eyes were enucleated and placed into cutting solution consisting of (mM): 125 Choline- $\mathrm{Cl}, 2.5 \mathrm{KCl}, 0.4 \mathrm{CaCl}_{2}, 6 \mathrm{MgCl}_{2}$, $1.25 \mathrm{NaH}_{2} \mathrm{PO}_{4}, 26 \mathrm{NaHCO}_{3}, 20$ D-glucose, on ice and saturated with $95 \%$ $\mathrm{O}_{2}$ plus $5 \% \mathrm{CO}_{2}$. The cornea was pierced with a $30 \mathrm{G}$ needle and the retina dissected out. The retina was then incubated for $2 \mathrm{~h}$ at room temperature in Ames medium (Sigma Aldrich; AUS) and 1.9g sodium bicarbonate (Sigma Aldrich; AUS) with either $1 \mathrm{mM}, 10 \mathrm{mM}$ or $100 \mathrm{mM}$, or 250 mM NAM (Sigma Aldrich; AUS) or control solution saturated with $95 \% \mathrm{O}_{2}$ and $5 \% \mathrm{CO}_{2}$. Retina were transferred to a submerged recording chamber on an upright microscope (Slicescope Pro 1000; Scientifica, UK). For all recordings, retina were perfused ( $2 \mathrm{ml} / \mathrm{min}$ ) with Ames medium saturated with $95 \% \mathrm{O}_{2}$ plus $5 \% \mathrm{CO}_{2}$ at $24{ }^{\circ} \mathrm{C}$, containing either $1 \mathrm{mM}, 10 \mathrm{mM}, 100 \mathrm{mM}$ or $250 \mathrm{mM}$ NAM or control $(0 \mathrm{mM})$ solution as per the previous incubation step. The center of the retina was located 
using infrared-oblique illumination microscopy with a $4 \mathrm{x}$ air objective (Olympus, Japan) and a CCD camera (IEEE 1394; Foculus, Germany) and retinal ganglion cells visualized using a $40 \times$ water-immersion objective (Olympus, Japan). Retinal ganglion cells with large cell bodies were chosen and their identity confirmed by the presence of AP firing and post-hoc biocytin immunohistochemistry (detailed below). Patch pipettes (6-9 M 2 ; GC150F-7.5; Harvard Instruments; USA) were pulled using a Flaming/brown micropipette puller (Model P-1000; Sutter instruments, USA) and filled with solution containing (mM): $115 \mathrm{~K}$-gluconate, $5 \mathrm{KCl}, 5$ EGTA, 10 HEPES, $4 \mathrm{Mg}$-ATP, $0.3 \mathrm{Na}$-GTP, and 7 phosphocreatine ( $\mathrm{pH}$ 7.3). Once whole-cell configuration was obtained, a holding current was injected to maintain a holding potential of approximately $-60 \mathrm{mV}$. Cells were excluded if a holding current greater than $-100 \mathrm{pA}$ was required to achieve a membrane potential of $-60 \mathrm{mV}$. Patch clamp recordings were performed using a PatchStar micromanipulator (Scientifica, UK) and Axon Multiclamp 700B patch-clamp amplifier (MDS, USA). Current clamp data were acquired using pClamp software (v10; MDS, USA) with a sampling rate of $100 \mathrm{kHz}$ and low pass Bessel filtered at $10 \mathrm{kHz}$ (Digidata 1440a; Axon). Action potential firing was recorded using an episodic protocol with the following current steps (0-200 pA in $10 \mathrm{pA}$ steps, $400 \mathrm{~ms}$ duration, $5 \mathrm{~s}$ inter sweep interval).

\subsection{Analysis of action potential firing}

Data were analyzed using AxoGraph X software (Berkeley, CA, USA). Action potentials were detected using an amplitude threshold of $50 \mathrm{mV}$ relative to pre-event baseline. Input-output curves were calculated by determining the number of action potentials generated for each injected current step. All action potential kinetics were analyzed for the first action potential at rheobase current, defined as the minimum current injection required to generate an action potential. Action potential threshold was defined as $30 \mathrm{mV} / \mathrm{s}$. Action potential amplitude was calculated relative to pre-event baseline. Action potential half-width was calculated at $50 \%$ of maximal action potential amplitude. The action potential rise-time was calculated as the time between $20 \%$ and $80 \%$ of the maximal action potential amplitude.

\subsection{Biocytin immunohistochemistry and morphological analysis}

Following the completion of recordings, retina were removed, fixed in $4 \%$ paraformaldehyde in $1 \mathrm{M}$ phosphate buffer overnight at $4{ }^{\circ} \mathrm{C}$ then washed three times for $5 \mathrm{~min}$ in $1 \mathrm{M}$ phosphate buffer. Retina were incubated overnight at room temperature in Alexa Fluor 488 streptavidin (S11223, ThermoFisher Scientific, USA). Retina were mounted using ProLong Gold Antifade Mountant (P36930, ThermoFisher Scientific, USA) and z-stack images were acquired on a Zeiss LSM 780 confocal microscope (Zeiss, Germany). Whole dendritic arbors were reconstructed using Imaris as above. Sholl analysis and dendritic field area measurements were generated in Imaris and soma diameter was measured on z-projections in Fiji (using the longest axis).

\subsection{Statistical analysis}

All statistical analysis was performed in R. Data were tested for normality with a Shapiro Wilk test. Normally distributed data were analyzed by Student's t-test or ANOVA (with Tukey's HSD). Nonnormally distributed data were transformed using squared transforms; data that remained non-normally distributed was analyzed by a KruskalWallis test followed Dunn's tests with Benjamini and Hochberg correction. Unless otherwise stated, $*=P<0.05, * *=P<0.01, * * * P<0.001$, NS $=$ non-significant $(P>0.05)$. For box plots, the center hinge represents the mean with upper and lower hinges representing the first and third quartiles; whiskers represent 1.5 times the interquartile range.

\section{Results}

3.1. Nicotinamide confers retinal ganglion cell neuroprotection in a range of glaucoma-relevant contexts

Nicotinamide is protective in a genetic mouse model (DBA/2J) that recapitulates features of a human pigmentary glaucoma and demonstrates an age-related decline in retinal NAD [5]. To further determine how nicotinamide (NAM) protects retinal ganglion cells (RGCs), we induced RGC degeneration in two separate glaucoma relevant insults in the absence of an age related effect: elevated IOP and optic nerve degeneration induced by axotomy. We first confirmed the neuroprotective effects of NAM in an inducible, acute rat model of glaucoma where the onset of high IOP is controlled and abrupt. Restriction of the anterior chamber drainage structures via magnetic microbead injection limits aqueous humor fluid outflow, producing significant ocular hypertension (OHT; elevated intraocular pressure) which is sustained for two weeks (Fig. 1A). NAD was significantly reduced in the retina and optic nerve of OHT animals at 14 days post-induction (Fig. 1B) (i.e. IOP-dependent, not age-dependent NAD decline). In vivo imaging using optical coherence tomography (OCT) demonstrated loss of the neuroretinal rim (representing intra-retinal retinal ganglion cell axons; Fig. 1C). Rats pre-treated with NAM $(200 \mathrm{mg} / \mathrm{kg} / \mathrm{d}$ dietary, representative of $2 \mathrm{~g} / \mathrm{d}$ for a $60 \mathrm{~kg}$ human [31]; with treatment continuing for the duration of OHT) did not demonstrate loss of the neuroretinal rim despite maintaining a similar IOP (Fig. 1C). OHT eyes demonstrated significant RGC loss as assessed by RBPMS + counting (an RGC specific marker in the retina, Fig. 1D). The average RGC nuclear diameter (RBPMS + DAPI nuclei) was also significantly decreased, suggesting that surviving cells were under considerable neurodegenerative stress (Fig. 1E) [32]. NAM treated OHT rats demonstrated significantly reduced RGC loss and nuclear shrinkage in a dose dependent manner (200, 400 , or $800 \mathrm{mg} / \mathrm{kg} / \mathrm{d}$ dietary, representative of 2,4 , or $8 \mathrm{~g} / \mathrm{d}$ for a $60 \mathrm{~kg}$ human [31]; Fig. 1E). These data support a neuroprotective role for NAM in the context of high IOP without an associated age-related NAD decline.

We next tested NAM protection in an acute, axon-specific injury using a retinal explant model $[12,13]$. Enucleation severs RGC axons in the optic nerve, and explants maintained in culture ex vivo, demonstrated a progressive RGC loss and nuclear shrinkage observed within $12 \mathrm{~h}$, with highly significant, reproducible RGC loss at 3 days ex vivo (Fig. 2A and B, Supplementary Fig. $1 \mathrm{~A}$ and B). This RGC loss and nucleus shrinkage is robustly prevented by NAM-supplemented media at a range of doses. Using this explant model we assessed whether protection extended to RGC axons and dendrites. RGC axons within the retinal nerve fiber layer (RNFL) demonstrated a significant increase in the number of varicosities, a metric of neurite degeneration, by 3 days $e x$ vivo, but with no change in average varicosity size (Fig. $2 \mathrm{C}$ and $\mathrm{D}$, Supplementary Fig. 1C and D). These changes were not observed in NAM treated retina, even by 3 days ex vivo, demonstrating significant protection against severe acute axon degeneration (Fig. $2 \mathrm{C}$ and D). At 3 days ex vivo RGCs had significant dendritic atrophy compared to control retina ( 0 days ex vivo), with reduced complexity (as assessed by Sholl analysis), total dendritic length, and dendritic field area. NAM treatment was highly effective, preserving dendritic morphology (Fig. 2E and F). Dendritic varicosities were significantly increased in density as a function of dendrite length and dendritic field area, indicating dystrophy and degeneration of dendrites. NAM at a range of doses prevented these changes (Fig. 2E and F). These data support previous findings on the protective effects of NAM, and extend its known effects to encompass neuroprotection across RGC compartments within the retina in additional animal models. 
A

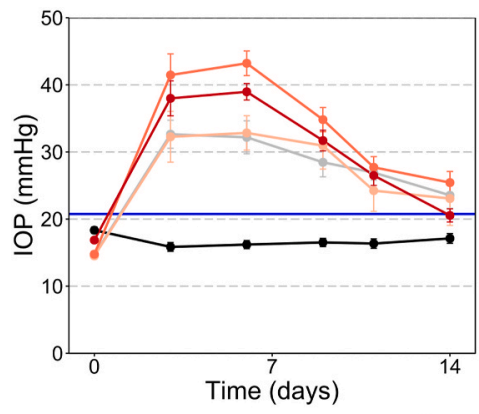

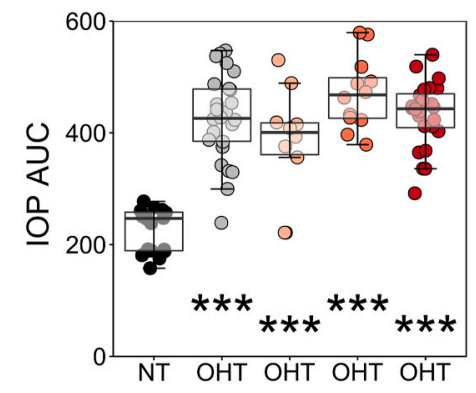

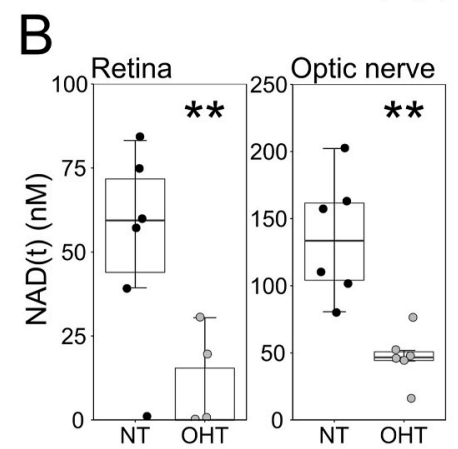

C
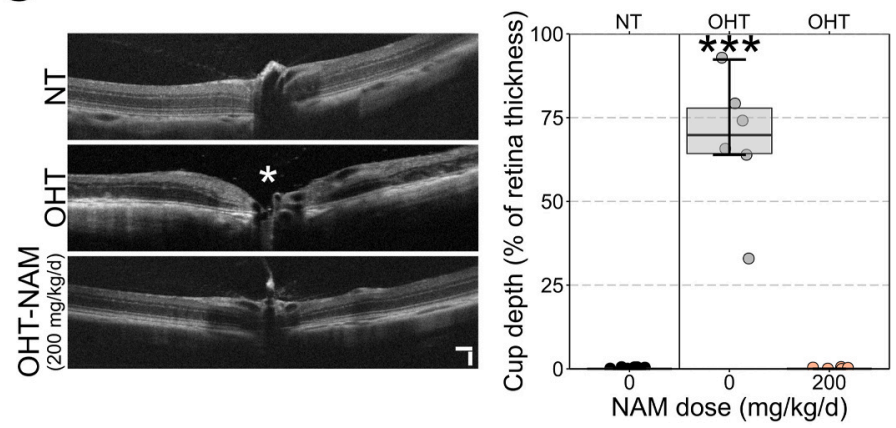

D

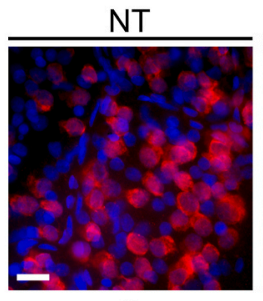

0

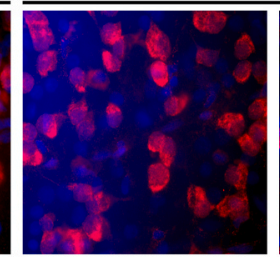

0

E
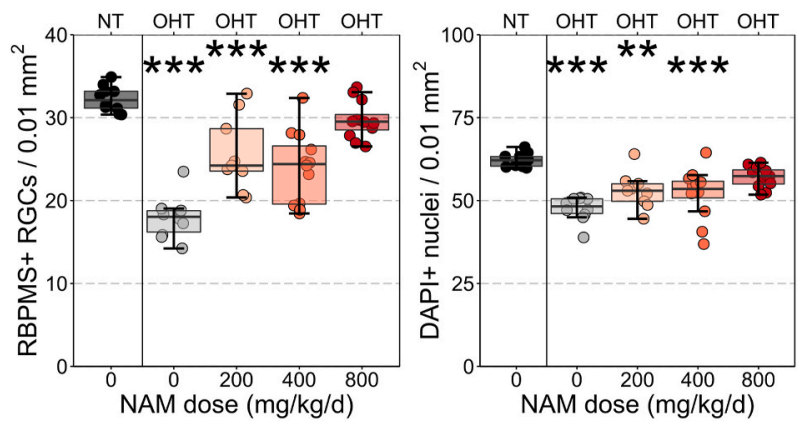

NAM dose $(\mathrm{mg} / \mathrm{kg} / \mathrm{d})$

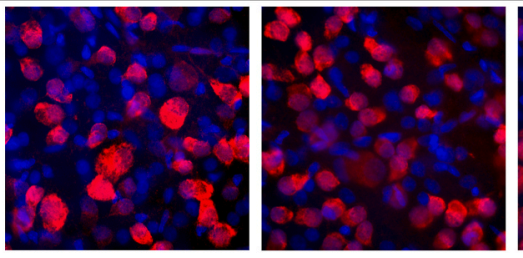

200

$\mathrm{AM}(\mathrm{mg} / \mathrm{kg} / \mathrm{d})$

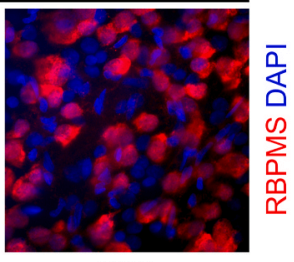

800

Fig. 1. Nicotinamide provides neuroprotection in a rat bead model of ocular hypertension. (A) Ocular hypertension (OHT; $n=26$ eyes) induced by the magnetic bead injection lead to a significant increase in IOP over control (NT; $n=20$ eyes), which persisted for 14 days (greater than 2 standard deviations of NT, blue line). Nicotinamide (NAM) treatment in OHT animals $(200,400,800 \mathrm{mg} / \mathrm{kg} / \mathrm{d} ; n=10,12$, and 24 eyes respectively) did not lower IOP (as assessed by area under the IOP curve). (B) Following 14 days of sustained OHT, NAD was significantly reduced in both the retina $(n=6)$ and optic nerve ( $n=6)$ as measured by luminometry-based assays. (C) In vivo OCT imaging demonstrated significant loss of neuroretinal rim following 14 days OHT ( $n=7$ eyes) compared to NT eyes ( $n=$ 9), which was absent in NAM treated rats ( $n=8$ eyes; measured as the cup depth relative to retinal thickness; loss denoted by white * on example image). (D) RBPMS (RGC specific) and DAPI labelling of the retina demonstrated a dose dependent, significant neuroprotection against RGC loss and RGC nuclear shrinkage at day 14 (E; $n=10$ NT retinas, 10 OHT, 9 OHT-NAM $(200 \mathrm{mg} / \mathrm{kg} / \mathrm{d}), 12$ OHT-NAM $(400 \mathrm{mg} / \mathrm{kg} / \mathrm{d})$, and 12 OHT-NAM $(800 \mathrm{mg} / \mathrm{kg} / \mathrm{d})) . S c a l e ~ b a r=100 \mu \mathrm{m}$ in C, $20 \mu \mathrm{m}$ in D. ** $P$ $<0.01,{ }^{* * *} P<0.001$. (For interpretation of the references to color in this figure legend, the reader is referred to the Web version of this article.)

\subsection{Nicotinamide alters metabolic profiles in normal, non-diseased retinal ganglion cells}

Given the importance of NAD in a wide array of metabolic processes and the potential of NAM as an interventional and prophylactic treatment in human glaucoma, we sought to determine whether NAM treatment alters RGC metabolic profiles under normal physiological conditions. We optimized a low molecular weight mass spectrometry platform to assess 73 essential low molecular weight metabolites across the RGC trajectory in normotensive rats (NT; normal IOP, no disease). We used bulk tissue (rat retina, optic nerve, superior colliculus (SC; the major RGC terminal in the rodent)) to avoid metabolic changes induced by cell sorting or tissue processing protocols. These data provide a novel metabolome across RGC compartments; dendrites, soma, and 
A
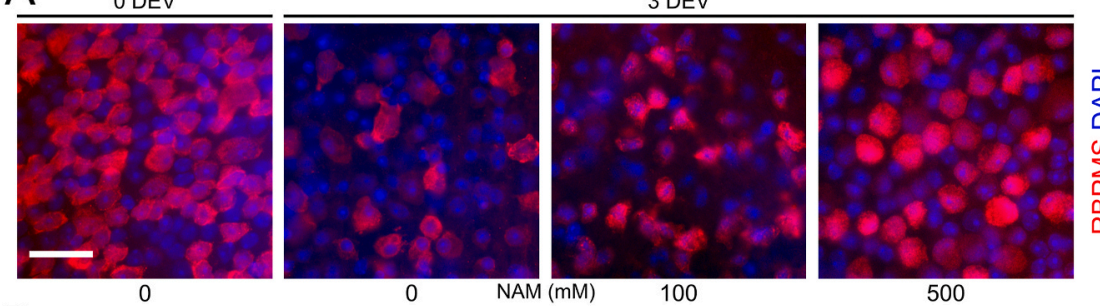

$\mathrm{B}$
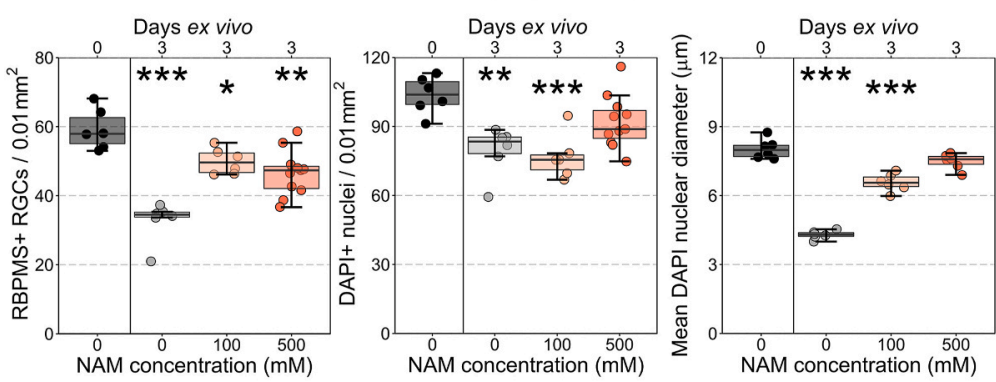

C
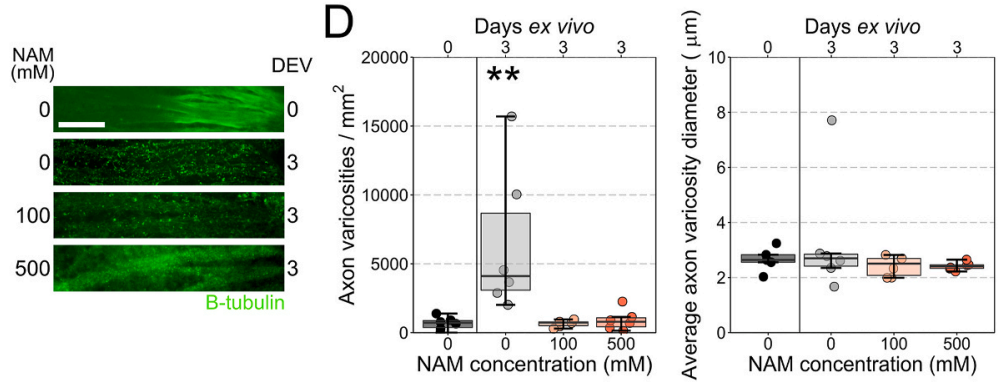

E NAM concentration ( $\mathrm{mM})$
NAM concentration (mM)

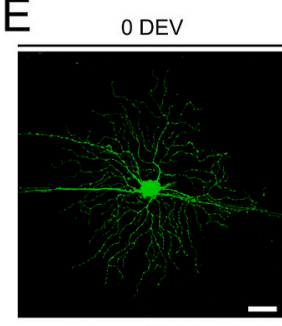

0

F
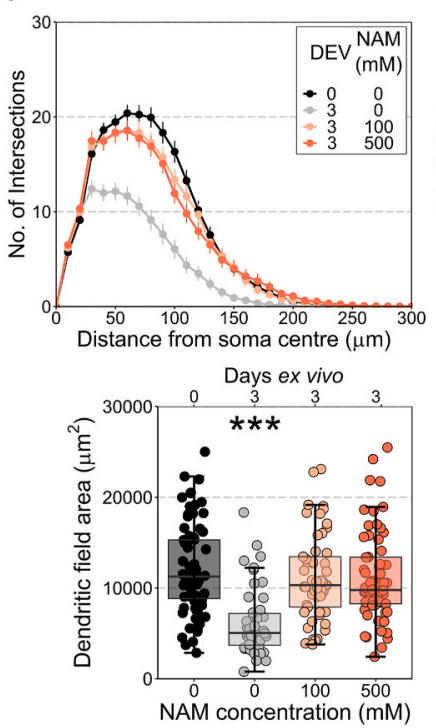

3 DEV

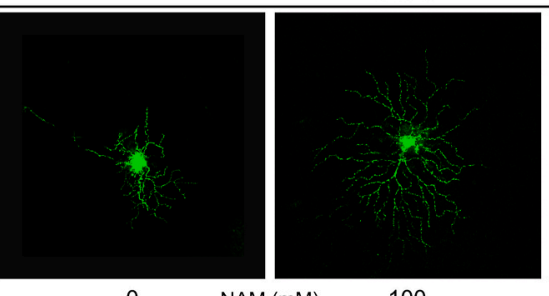

NAM (mM) 100
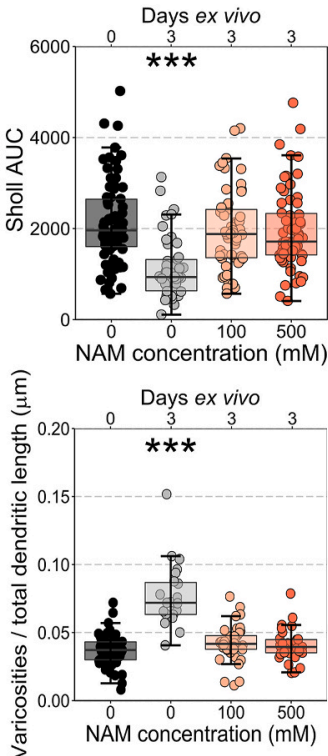

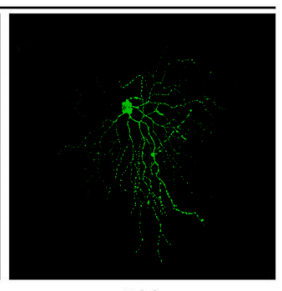

500

Fig. 2. Nicotinamide protects against axonal and dendritic degeneration. (A) Retinal axotomy explant results in significant RGC death and nuclear shrinkage, at 3 days ex vivo (DEV) with robust protection provided by NAM treated media (B; $n=$ six 0 DEV retinas, six 3 DEV untreated, six 3 DEV-NAM (100 mM), eleven 3 DEV-NAM (500 mM)). (C) $\beta$-tubulin labelling reveled a significant increase in axonal varicosities within the retinal nerve fiber layer at $3 \mathrm{DEV}$, which were robustly protected against by NAM (D; $n=$ six 0 DEV retinas, six 3 DEV untreated, six 3 DEV-NAM (100 mM), six 3 DEV-NAM (500 mM)). (E and F) At $3 \mathrm{DEV}$ significant changes to the complexity of the dendritic arbor were apparent (E; example RGCs labelled by DiOlistics). At $3 \mathrm{DEV}$ there was a loss of branch density (reduction in Sholl AUC), a reduction in dendritic length and field area. NAM treated media provided significant protection against these neurodegenerative features $(n=$ 75 RGCs from ten 0 DEV retinas, 46 RGCs from twelve 3 DEV untreated, 53 RGCs from twelve 3 DEV-NAM (100 $\mathrm{mM}), 62$ RGCs from twelve 3 DEV-NAM (500 mM)). At 3 DEV dendrites display an increased density of varicosities, which was protected against by NAM (F; $n=55$ RGCs from 0 DEV retinas, 23 RGCs from 3 DEV untreated, 38 RGCs from 3 DEV-NAM (100 mM), 32 RGCs from 3 DEV-NAM $(500 \mathrm{mM}))$. Scale bar $=20 \mu \mathrm{m}$ in A, $50 \mu \mathrm{m}$ in C and $\mathrm{E}$. $* P<0.05,{ }^{* *} P<0.01,{ }^{* * *} P<0.001$. 
unmyelinated axons (retina), myelinated axons (optic nerve), and terminal arbors (SC) (Supplementary Dataset 1 and 2; raw and normalized data). The normal rat metabolome varies significantly by these selected regions, with all 73 metabolites differentially abundant across tissues. Unsupervised hierarchical clustering (HC) separated samples into discrete groups that matched the different tissues, confirming globally distinct metabolic profiles (Supplementary Fig. 2A). Metabolites were most highly abundant in the optic nerve, followed by the SC and retina respectively. Correlation of all individual metabolites for all samples demonstrated that the SC had the highest intra-tissue correlation (Fig. 3A). In order to identify distinct metabolic signatures of the discrete tissues we used principle component analysis (PCA). Unsupervised PCA separated samples into discrete tissue groups supporting the HC data with PC1 describing the vast majority of variation $(95.3 \%$; Fig. 3B). Creatine, hypotaurine, 2-aminobutyric acid, glycerophosphocholine, and cysteine were the highest contributing loading factors in to PC1 demonstrating that relative abundance remained the largest determinant of tissue type even after scaling to reduce its effects (Supplementary Fig. 2B and C). We specifically queried how NAD, NADH, and NAM vary across these tissues. NAD was higher in retina and optic nerve than in the SC, but NADH was much more abundant in the optic nerve. This results in a lower NAD:NADH ratio in the optic nerve. NAM was relatively low in the retina compared to both the optic nerve and SC (Fig. 3C).
NT rats treated with NAM 10 days prior to metabolomic analysis demonstrated a small but statistically significant reduced IOP of -1.2 $\mathrm{mmHg}$ (the physiological impact of which is likely to be limited) (Fig. 4A). (It is important to note that NAM treatment did not affect IOP in the context of OHT; Fig. 1A.) HC of individual samples demonstrated that NT-NAM treated retina were largely similar to normal NT retina, but there was a clear distinction in metabolic profile in the optic nerve and SC (Fig. 4B). PCA demonstrated that NAM induced changes were not sufficient to drive group distinction (Fig. 4B). NAM treatment resulted in 9 changed metabolites $(11 \%)$ in the retina, 24 in the optic nerve (30\%), and 5 in the SC (6\%) (Fig. 4C-F, Supplementary Dataset 3). Increased $\mathrm{NAD}, \mathrm{NADH}$, and threonine were common to all tissues, with glyceric acid also increased in both retina and optic nerve (Fig. 4G, Supplementary Fig. 3). These metabolites alone were sufficient to distinguish NT and NT-NAM samples by HC (Fig. 4G). NAM was only increased in the optic nerve, suggesting that conversion to NAD may be saturated in the optic nerve at this dose (Supplementary Fig. 3). The NAD:NADH ratio was only significantly altered in the optic nerve ( $40 \%$ increase), as NAD was increased to a greater proportion than NADH $(\sim 2.4$ fold compared to $\sim 1.7$ fold), suggesting a larger pool of free NAD (Supplementary Fig. 3). Pathways analysis revealed that NAM induced changes are predicted to significantly impact nicotinate and nicotinamide metabolism across the retina and optic nerve (Fig. 4H, Supplementary Dataset 4). Arginine biosynthesis is predicted to be affected in the retina,
A

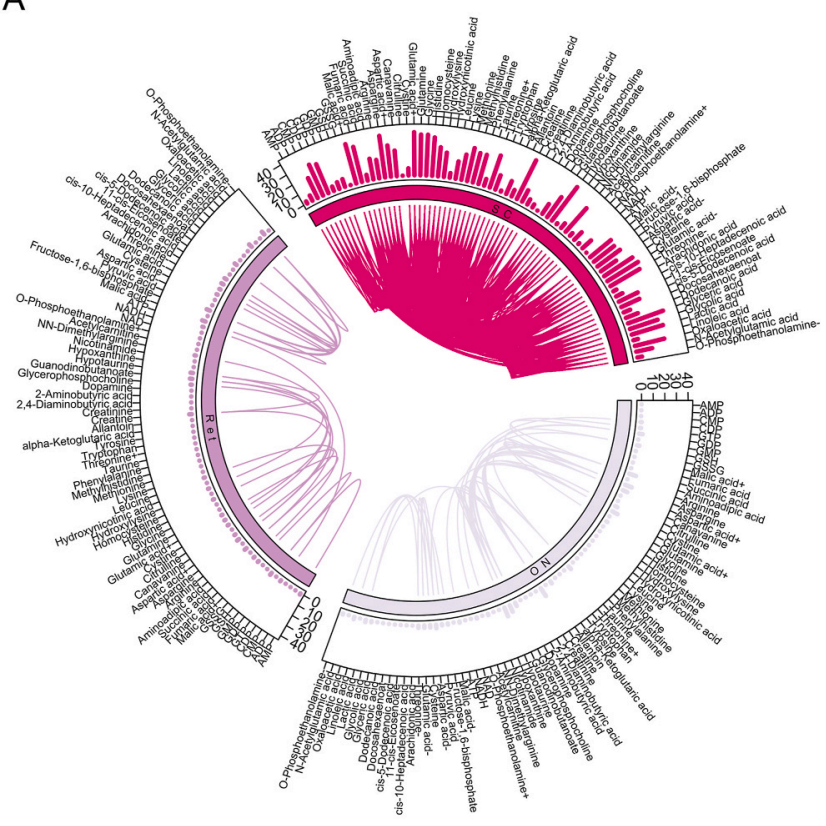

B

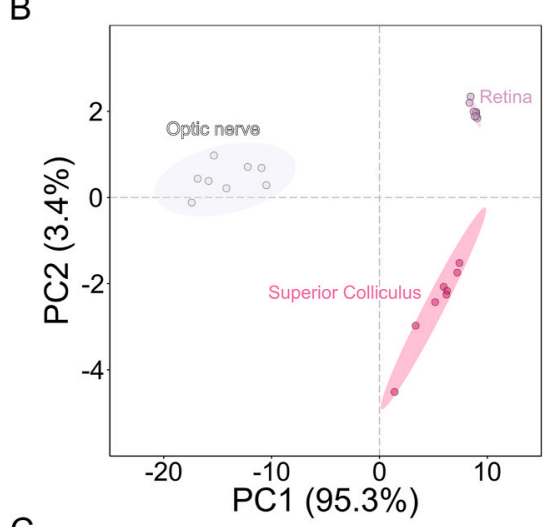

C
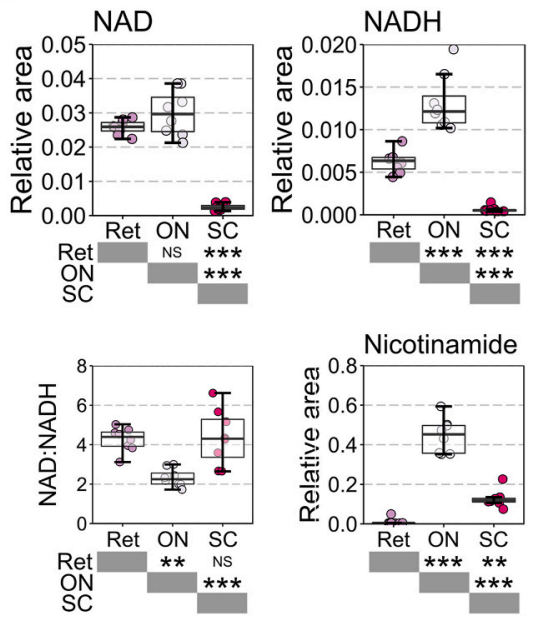

Fig. 3. Metabolic profiles of retinal ganglion cell related tissues. (A) Seventy three low molecular weight metabolites could be reliably detected in retina $(n=8)$, optic nerve $(n=8)$ and superior colliculus (SC; $n=8$ hemispheres) of Brown Norway rats. Within tissue correlation of metabolites is demonstrated by a circus plot with linkers between metabolites. The number of significantly correlated metabolites is shown as a bar graph in the outer circle. The SC (pink) showed the greatest degree of within tissue correlation of metabolites. (B) Tissue differences were explored by principle component analysis (PCA) which revealed a clear distinction of tissues predominantly along one component (PC1). (C) NAD and related metabolites NADH and nicotinamide were compared between tissues. The optic nerve was most abundant in NAD, NADH, and nicotinamide but had the lowest NAD:NADH ratio, whilst the retina was comparatively low in nicotinamide, and the SC comparatively low in NAD and NADH. ${ }^{* *} P<0.01,{ }^{* * *} P<0.001$, NS $P>0.05$. (For interpretation of the references to color in this figure legend, the reader is referred to the Web version of this article.) 
A

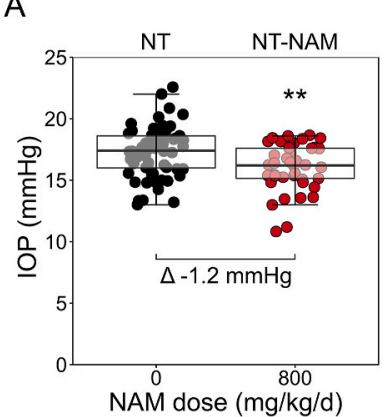

C

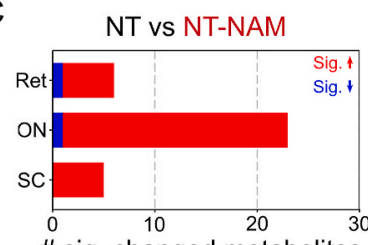

\# sig. changed metabolites

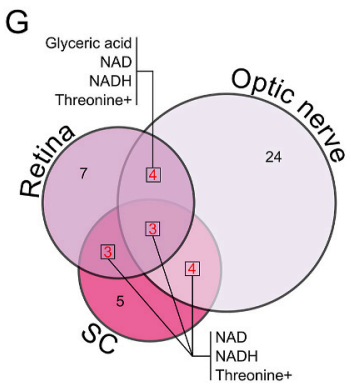

$\mathrm{H}$

B
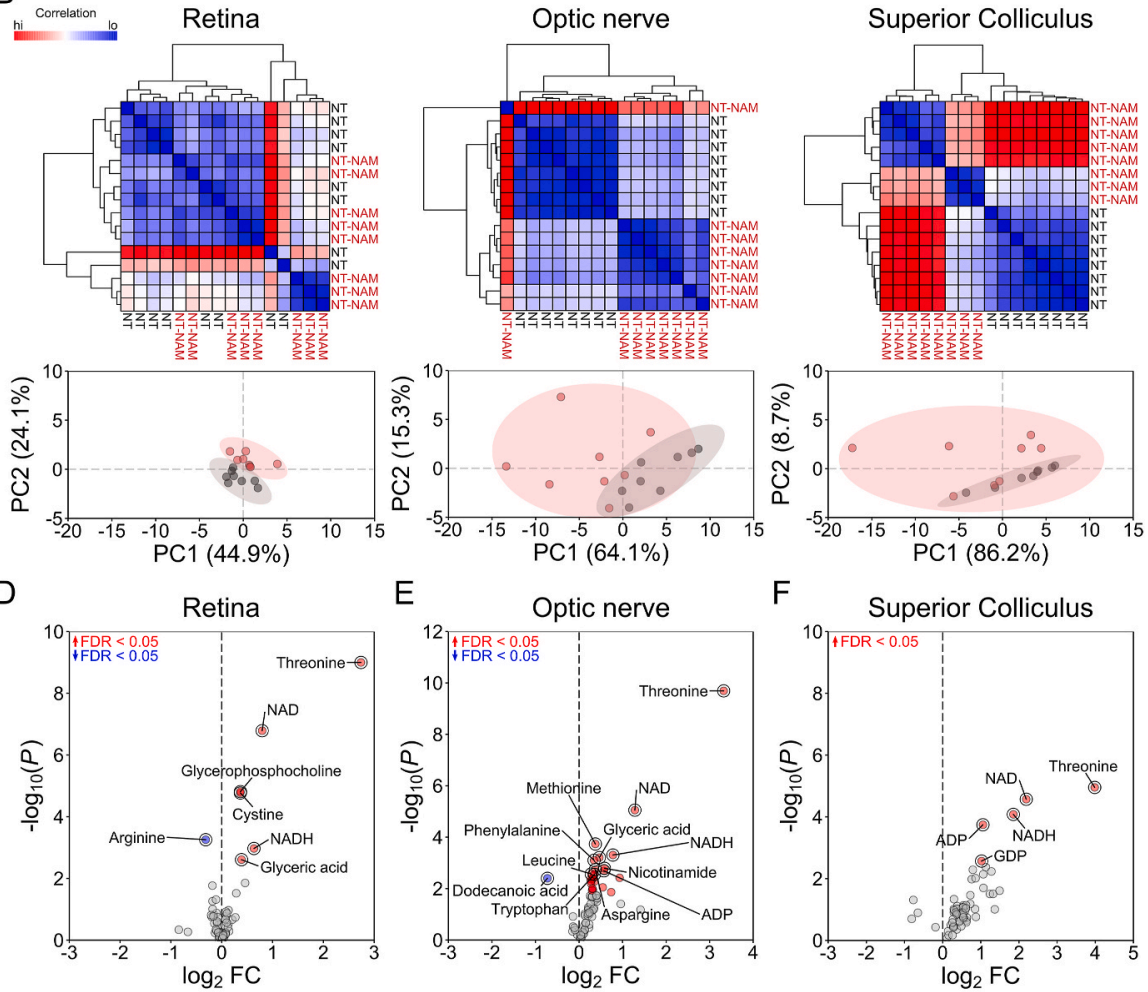

E Optic nerve

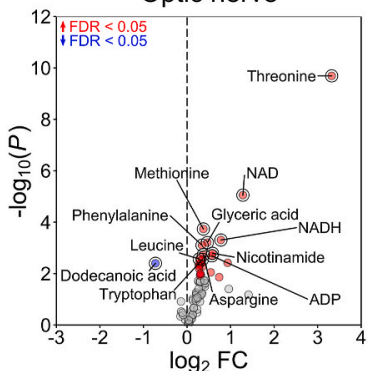

F
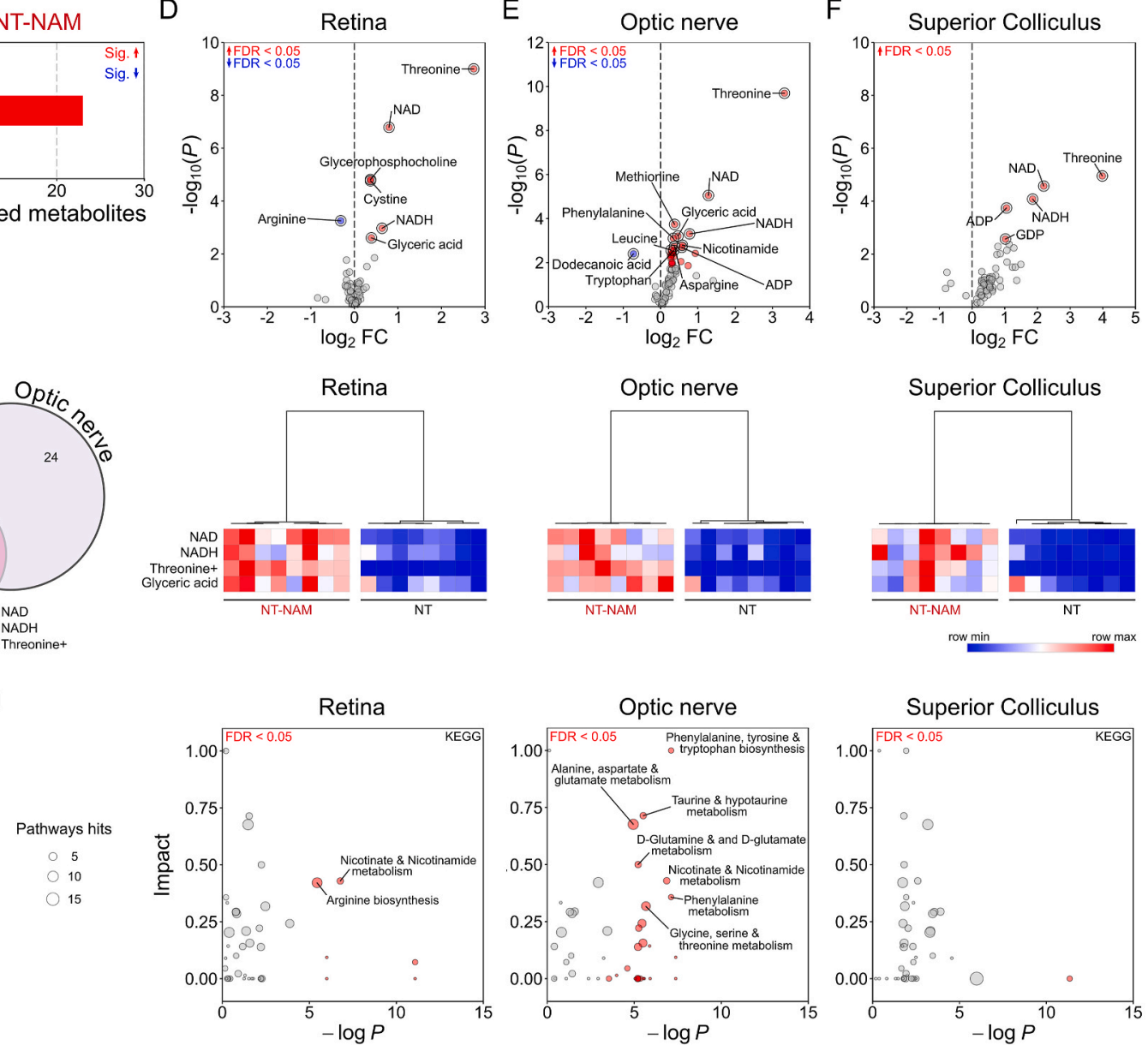

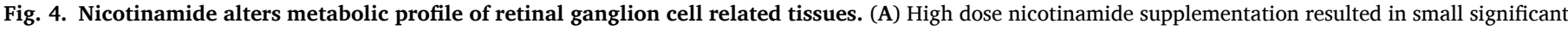

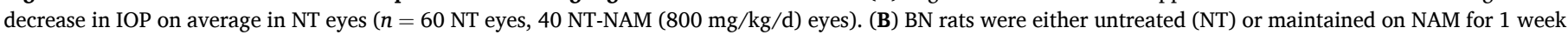

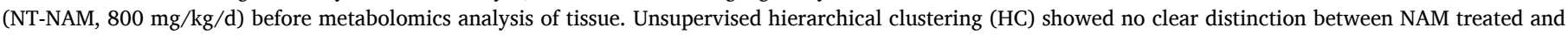

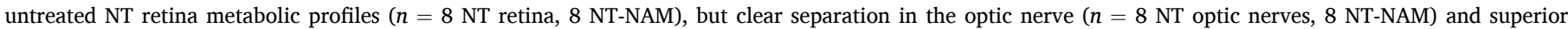

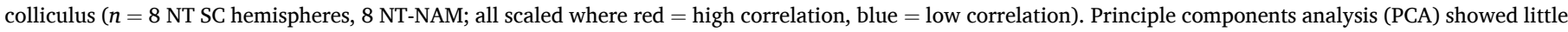

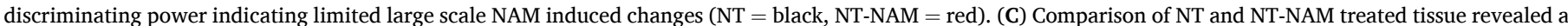

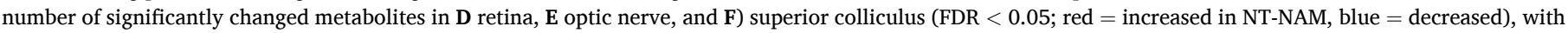

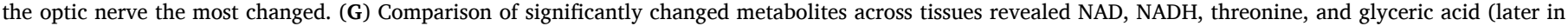

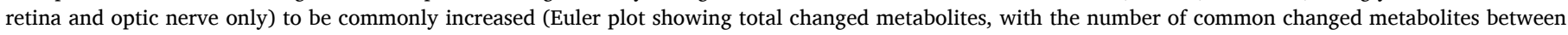

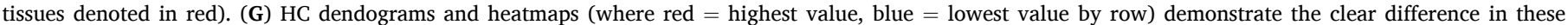

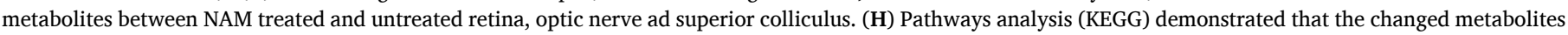

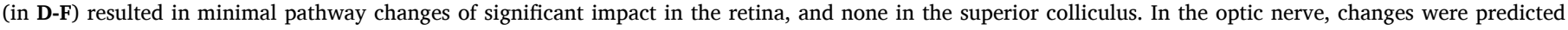

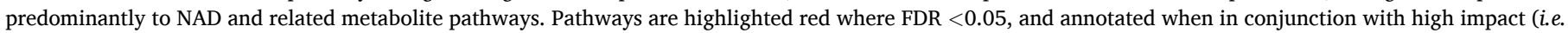

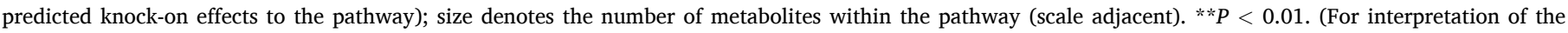
references to color in this figure legend, the reader is referred to the Web version of this article.) 
and phenylalanine, tyrosine and tryptophan biosynthesis, taurine and hypotaurine metabolism, alanine, aspartate and glutamate metabolism, D-glutamine and D-glutamate metabolism, and glycine, serine and threonine metabolism are predicted to be altered in the optic nerve. In these pathways, significant impact was attributed predominantly to NAM and NAD or amino acids L-threonine, L-glutamate, L-aspartate, and phenylalanine. There were no predicted pathway changes in SC dataset (Fig. 4H). When cultured in NAM, dissociated neurons from retina, optic nerve, and cortex produce increased NAD over untreated cells demonstrating the ability to rapidly and directly metabolize NAM to NAD without the need for first pass metabolism (Fig. 5).

\subsection{Nicotinamide protects against metabolic disruption following ocular hypertension}

In order to determine the NAD and metabolic changes that may precede RGC death we performed metabolomics following 3 days of OHT, a time point at which IOP is high but no detectable RGC loss has occurred [11]. The retinal metabolome is significantly altered at this 3 day time point. HC revealed heterogeneity across and within conditions, but with a division between samples such that NAM treated eyes were largely grouped and distinguished (Fig. 6A). In the retina OHT led to 25 changed metabolites relative to NT controls (24 increased, 1 decreased). Notable changes were increased alpha-ketoglutaric acid, homocysteine, threonine, NAD, and glycerophosphocholine, while creatinine was the sole reduced metabolite (Fig. 6B). Comparison of OHT-NAM treated retinas to NT-NAM (thus excluding normal NAM induced changes) revealed that NAM treatment completely prevented any OHT induced metabolite changes (Fig. 6B). NAD remained high in OHT-NAM retinas (Supplementary Fig. 4A). Pathway analysis predicted changes to alanine, aspartate and glutamate metabolism, D-glutamine ad D-glutamate metabolism, arginine biosynthesis, glyoxylate and dicarboxylate metabolism, and histidine metabolism (Fig. 6C).

In the optic nerve, HC revealed discrete untreated and NAM groups (Fig. 6D). No metabolites were significantly changed in OHT relative to NT (Fig. 6E). In OHT-NAM, 24 metabolites were significantly lower than in NT-NAM. Comparison of OHT-NAM to untreated OHT and NT demonstrated only 2 and 4 changed metabolites respectively (Fig. 6F). Collectively, these data suggest that the NAM induced metabolic profile change exhibited in normal tissue is lost during OHT. Comparison of NAM changes in NT (NT vs. NT-NAM) and OHT (NT-NAM $v s$. OHT-NAM)

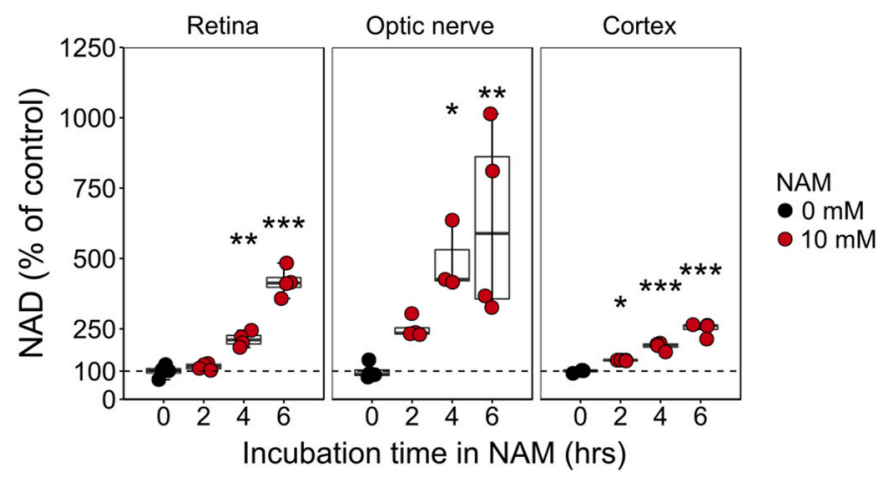

Fig. 5. Nicotinamide can be rapidly metabolized to NAD by retina, optic nerve, and brain tissue. Luminometry-based NAD assays on retina, optic nerve and cortex incubated with NAM showed that it can be converted to NAD within hours, increasing with increased exposure, suggesting an increase in the NAD pool ( $n=4$ retinal replicates where each is composed of 2 pooled retinas, for each time point a sample was taken from each replicate; $n=4$ optic nerve replicates where 8 optic nerves were divided in to $1 \mathrm{~mm}$ segments to give 16 independent samples for each time point; $n=4$ cortex replicates where each replicates is a single cortex hemisphere and for each time point a sample was taken from each replicate). Optic nerve demonstrated the largest increase over control (untreated optic nerve) supporting the metabolomics observations. optic nerve demonstrated 16 commonly changed metabolites that reflect NAM specific changes (Fig. 6G). HC using only these 16 metabolites was sufficient to separate individual NAM treated groups from untreated groups. Comparison of these changed metabolites relative to NT demonstrated marked increases in NT-NAM only, with OHT-NAM retuned to NT levels across all metabolites (with the exception of NAD and threonine) (Fig. 6G). Crucially, NAD, NADH, and NAM remained higher in OHT-NAM optic nerves than in untreated OHT optic nerves (Supplementary Fig. 4B). As high NAD is axon protective [33], then this suggests that there is likely to be a longer term neuroprotection to the optic nerve afforded by NAM. However, this will need to be definitively tested at later disease time points than those explored here.

In the SC, NAM treated conditions were clearly distinguished by HC (Fig. 6H). During OHT 6 metabolites declined relative to NT controls, of which 5 of these changes were prevented in OHT-NAM (Fig. 6I). NAM treatment in OHT was sufficient to replete NAD in the SC, with a diminished increase over normal NT levels relative to NT-NAM (Supplementary Fig. 4C). The small number of metabolite changes in the SC were insufficient to drive any predicted pathways changes.

Comparison of changes in OHT across tissues revealed 5 commonly changed metabolites in the retina and SC (Fig. 6J). Of these, all were changed in opposing directions, demonstrating a concomitant increase in the retina and decrease in the SC. These data support a hypothesis in which RGC metabolism is altered early during glaucoma pathogenesis and that this is largely prevented or buffered by oral NAM administration.

\subsection{Nicotinamide protects retinal ganglion cells from metabolic crisis}

We have previously demonstrated dysregulated gene expression in RGCs across multiple metabolic pathways [5], including widespread upregulation of genes encoding oxidative phosphorylation (OXPHOS) proteins. Given that genetic changes coincide with mitochondrial cristae loss and increased cytochrome $c$, it can be hypothesized that these may reflect an upregulated transcriptional response in order to increase mitochondrial OXPHOS capacity. Gene expression in axotomized retina demonstrated a reduction in the mitochondrial-RNA:nuclear-RNA ratio suggesting a loss of mitochondrial capacity or a reduced number of mitochondria (Fig. 7A). We queried whether the ratio of ATP:ADP was reduced in OHT, which would indicate a switch towards glycolysis from OXPHOS, and how this may be affected by NAM treatment. No significant changes in this ratio were detected in the retina or optic nerve at 3 days in the rat OHT model. In the SC, the ATP:ADP ratio was significantly reduced in OHT and this was prevented by NAM treatment (Fig. 7B). Since within condition variability was high, which may reflect mixed cell type-specific responses, we analyzed OXPHOS and glycolysis in purified mouse RGC cultures with NAM supplementation. Oxygen consumption rate was significantly increased in cells supplemented with 50 and $500 \mu \mathrm{M}$ NAM, with the effect diminished at higher concentrations. No significant change in extracellular acidification rate was detected (Fig. 7C). These findings suggest that NAM supplementation increases RGC OXPHOS capacity. We therefore tested the ability of NAM to protect RGCs against OXPHOS inhibition using an intravitreal rotenone model (Complex I inhibition which results in the rapid degeneration of RGCs). Intravitreal injection of rotenone induced significant RGC death within 1 day, which was significantly reduced by NAM supplementation (Fig. 7D and E). Thus, in the absence of axon degenerative stress, nicotinamide can provide a robust neuroprotection against mitochondrial stress.

\subsection{Nicotinamide increases mitochondrial size and motility}

Mitochondrial remodeling and/or loss is a common feature of neurodegenerative disease which may drive neuronal metabolic dysfunction. We directly assessed changes to mitochondrial morphological integrity after 14 days of OHT in the rat. TOMM20 labelled 


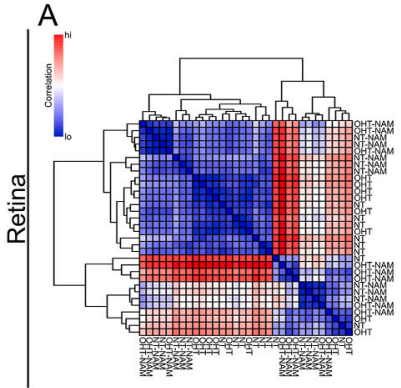

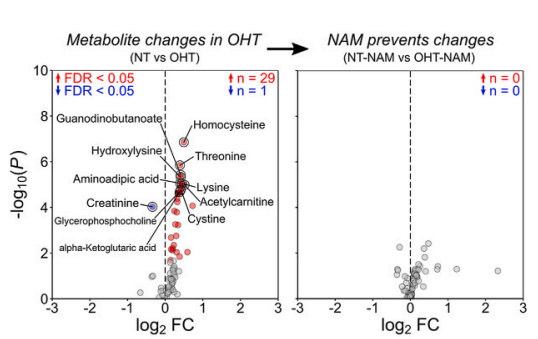
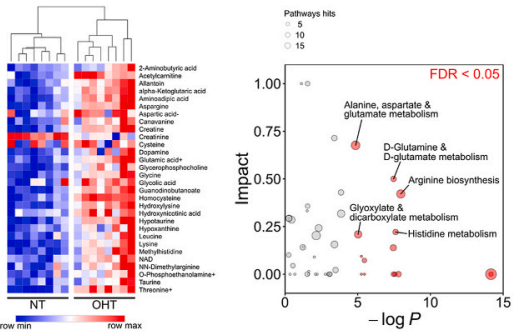

G

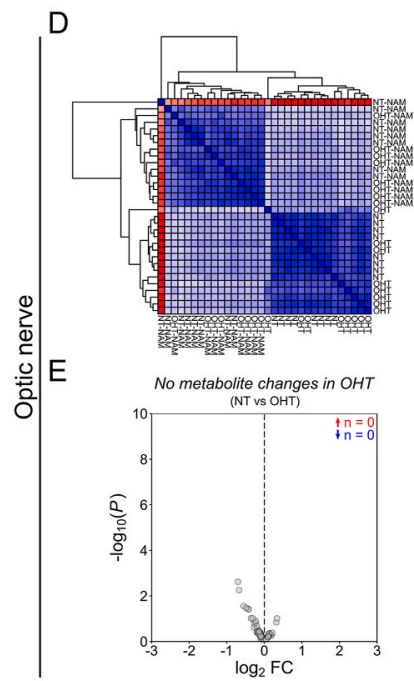

F
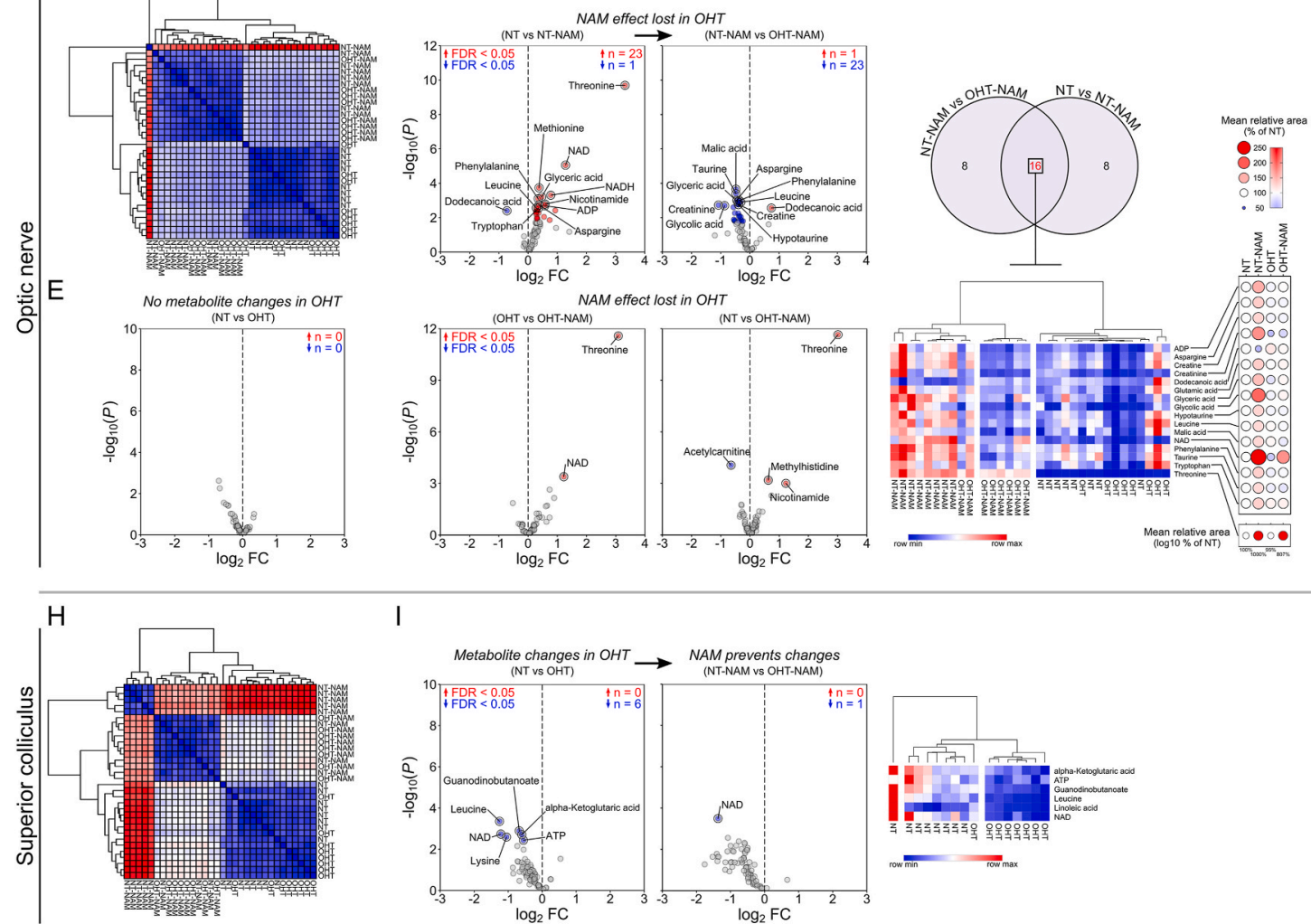

I

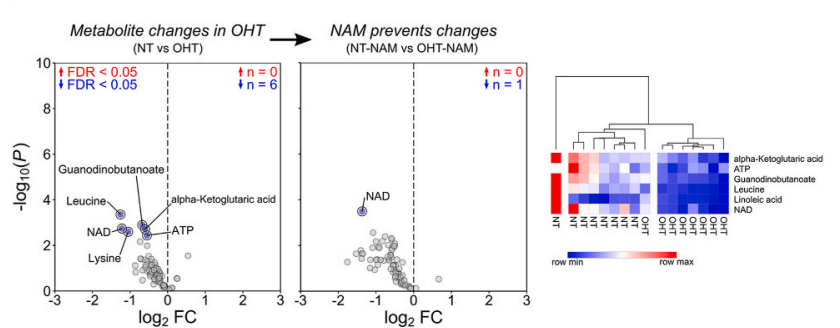

J

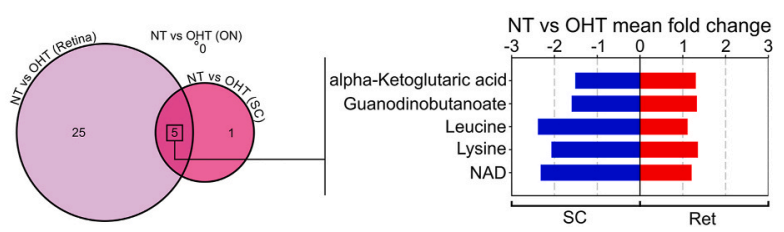

(caption on next page) 
Fig. 6. Nicotinamide prevents metabolic disruption. The metabolic profiles of BN rats following 3 days of OHT (prior to detectable neurodegeneration) were compared to NT controls, and the effects of pretreatment with NAM $(800 \mathrm{mg} / \mathrm{kg} / \mathrm{d})$ explored $(n=8$ retinas, optic nerves and superior colliculus hemispheres for each condition). (A) In the retina, unsupervised hierarchical clustering (HA) demonstrated that samples largely clustered based on exposure to NAM, but with heterogeneity across conditions (heatmap scaled where red $=$ high correlation, blue $=$ low correlation). (B) OHT induced a number of metabolic disturbances with a number of significantly increased metabolites (NT vs OHT; FDR $<0.05$; red $=$ increased in NT-NAM, blue $=$ decreased; further shown in HA dendogram and heatmap; red = highest value, blue = lowest value by row); these changes were completely absent in NAM treated retinas (NT-NAM vs OHT-NAM; which controls for NAM specific effects). (C) Pathways analysis (KEGG) demonstrated a number of potentially effected pathways (pathways are highlighted red where FDR $<0.05$, and annotated when in conjunction with high predicted impact, size denotes the number of metabolites within the pathway). (D) In the optic nerve, HA revealed clear distinction between NAM treated and untreated samples, irrespective of disease grouping. (E) No metabolites were significantly altered compared to NT following 3 days of OHT (NT vs OHT). (F) While NT-NAM treated optic nerves demonstrated a number of significantly changed metabolites over NT nerves, these effects were lost under OHT as demonstrated by the reversal of a number of these changes (NT-NAM vs OHT-NAM) and the similar profiles of OHT-NAM optic nerves to both OHT and NT nerves (OHT vs OHT-NAM, NT vs OHT-NAM respectively). (G) Comparison of NT-NAM vs OHT-NAM changes against NT vs NT-NAM changes, demonstrated 16 commonly changed metabolites (Euler plot showing total changed metabolites, with the number of common changed metabolites between comparisons denoted in red). HA of these metabolites separated individual NAM treated groups from controls, and plotting the mean abundance of these relative to NT control further highlights NAM specific changes that were reversed under OHT. (H) In the superior colliculus, HA largely distinguished individual groups, suggesting well defined metabolic profiles per condition. (I) In the SC, OHT resulted in a significant decrease of metabolites (NT vs OHT), which was prevented by NAM treatment (NT-NAM vs OHT-NAM). NAD in the SC was lower in OHT-NAM treated than NT-NAM treated (but remained above NT and OHT control levels; Supplementary Fig. 4). (J) Comparison of OHT changes relative to NT in the retina, ON and SC revealed that 5 metabolites were commonly changed, where increases in the retina were mirrored by decreases in the superior colliculus (Euler plot showing total changed metabolites, with the number of common changed metabolites denoted; for mean fold change plot, red $=$ increase, blue $=$ decrease). ${ }^{*} P<0.05$, ${ }^{* *} P<0.01,{ }^{* * *} P<0.001$. (For interpretation of the references to color in this figure legend, the reader is referred to the Web version of this article.)

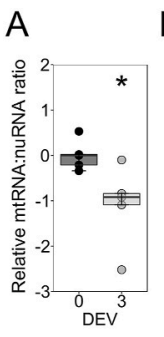

B

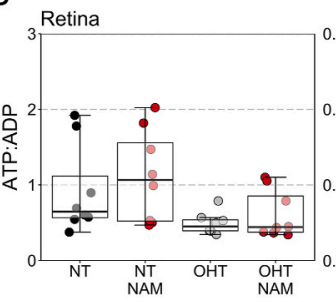

D

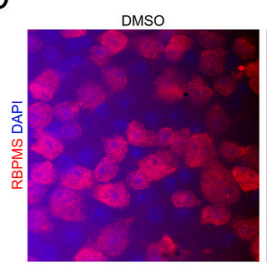

Optic nerve

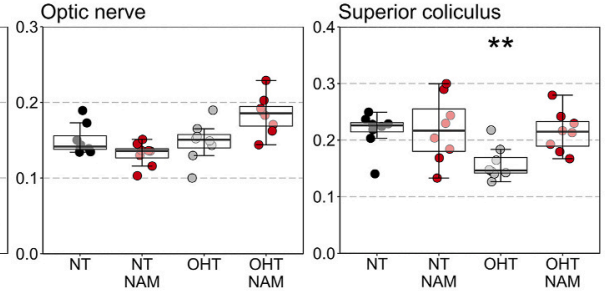

C

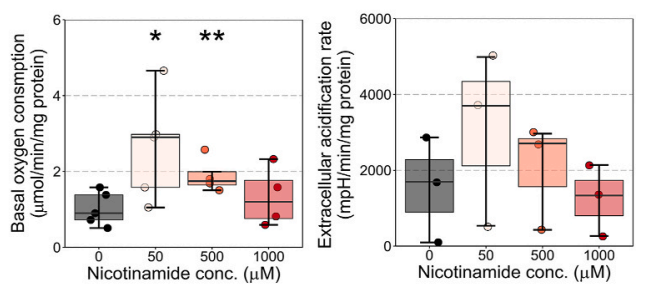

E
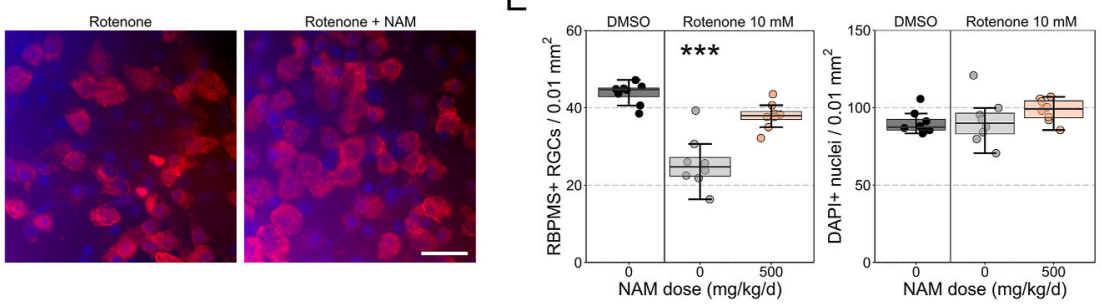

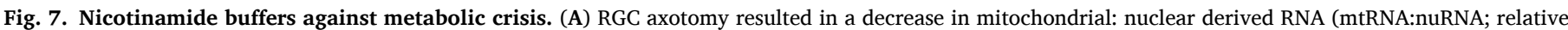

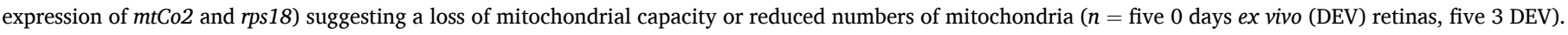

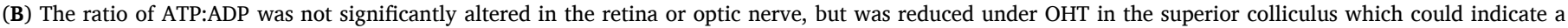

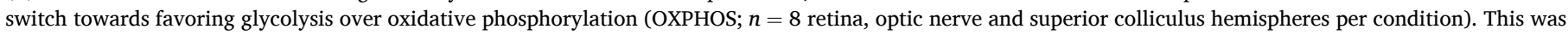

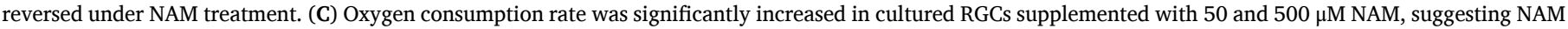

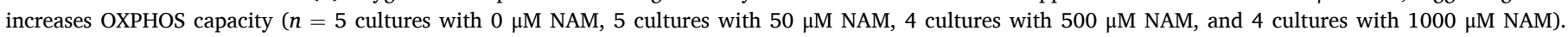

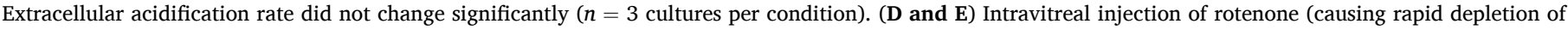

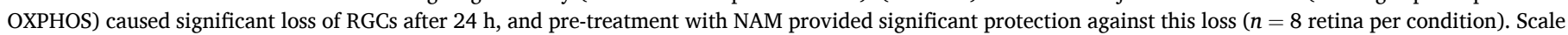
bar in $\mathrm{D}=20 \mu \mathrm{m} * P<0.05,{ }^{*} * P<0.01,{ }^{*} * P<0.001$.

mitochondria were imaged by super-resolution Airy confocal microscopy and reconstructed (Fig. 8A). Mitochondrial volume and surface area were significantly reduced in the ganglion cell layer (GCL) and RNFL (comprising RGC soma and axons and other retinal neurons/glia) of OHT rats (Fig. 8B). NAM treatment partially protected from these mitochondrial degenerative changes. In OHT rats, mitochondrial volume and surface area were significantly increased in the IPL (comprising RGC dendrites and interneuron neuropil), and further increased in OHTNAM treated rats (Fig. 8C). These different changes between retinal layers may be representative of the various cell types that make up these layers, their neuronal networks, and/or how they respond to OHT. To determine RGC specific mitochondrial responses to injury and NAM, we utilized a novel mitochondrial reporter mouse where YFP is expressed under a rat Eno2 promoter (neuron specific) and localized to mitochondria via a Cox8a gene-targeting signal fused to the YFP N-terminus (neuronal expression shown in Supplementary Fig. 5). In this strain (designated MitoV; for visual system tissue), YFP expression is RGC specific in the inner retina, allowing the assessment of mitochondria specifically in RGCs (with expression in a subset of bipolar cells and photoreceptor outer segments which can be optically dissected; Supplementary Fig. 5A-C). We hypothesized that physical axonal damage and mitochondrial stress work in concert to drive vision loss in glaucoma. To assess each of these in an isolated context, we induced RGC axotomy (uncoupling the RGC from the terminal visual thalami) or acute, severe mitochondrial dysfunction (intravitreal rotenone injection). Using the retinal explant model to induce an RGC specific injury, we assessed mitochondrial morphology in the GCL/RNFL (Fig. 9A). At $12 \mathrm{~h}$ ex vivo mitochondrial volume and surface area were significantly increased. NAM supplementation of the media exaggerated the increase in mitochondrial volume and surface area (Fig. 9B). Intravitreal injection of rotenone resulted in a significant reduction of mitochondrial volume and surface area in the IPL at 1 day post injection. NAM 
A

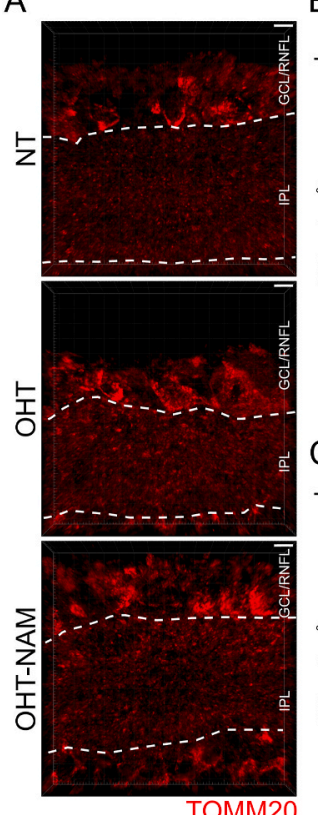

TOMM20

B

Ganglion cell layer / retinal nerve fibre layer (GCL / RNFL)
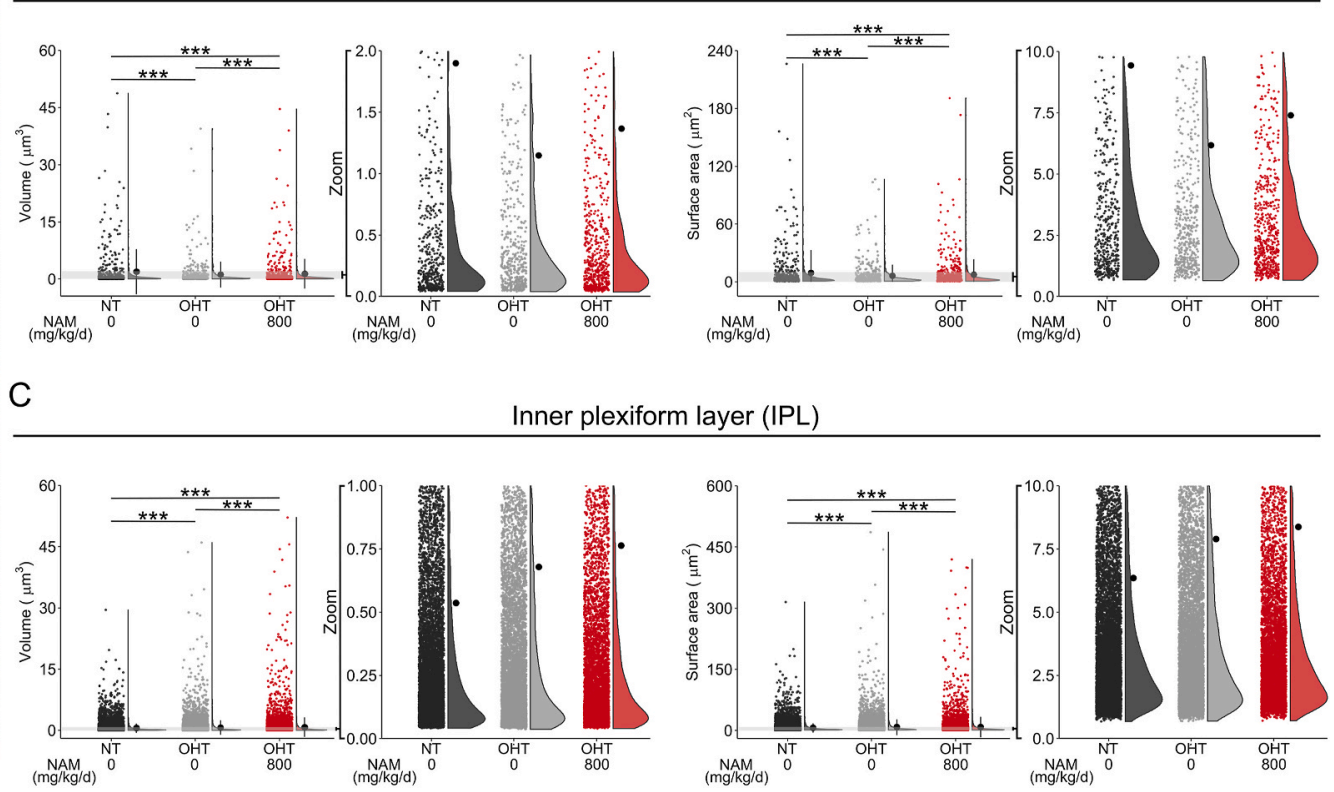

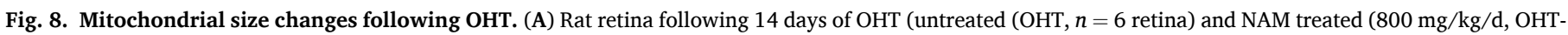

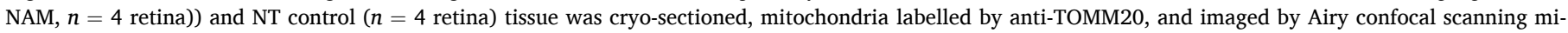

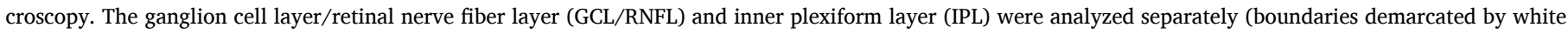

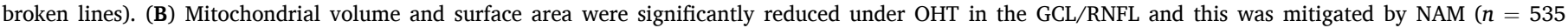

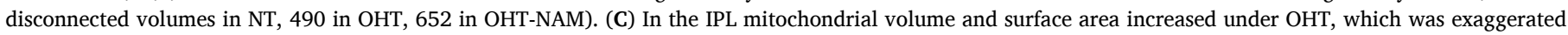

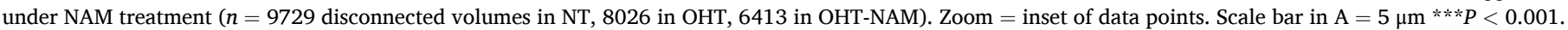

supplementation was unable to prevent these morphological changes even though NAM treatment was grossly neuroprotective (Fig. 9C and D). These data suggest that even across a range of insults, mitochondria increase or maintain their size when NAM is present. However, these changes may reflect the tissue culture conditions (e.g. $\mathrm{Ca}^{2+}$ buffering deficits or deviation from normoxia in the axotomy model) or the abrupt complete physiological loss of mitochondrial Complex I function (in the rotenone model) which are exaggerations of the intrinsic RGC injury responses during glaucoma pathogenesis.

In order to differentiate NAM-induced and insult-induced mitochondrial changes we administered NAM in the diet of uninjured control MitoV mice for 1 week prior to fixation and imaging (Fig. 9E). RGC mitochondria in the GCL/RNFL increased in volume and surface area compared to untreated controls, suggesting that the increase in volume and surface area observed in the NAM treated injury paradigms were NAM specific responses (Fig. 9F). The presence of larger mitochondria prior to damage may therefore explain the maintenance of mitochondrial volume and surface area which was observed in the rat GCL/RNFL in NAM treated injuries and the exaggerated mitochondrial expansion seen in the rat IPL. In vivo imaging of MitoTracker labelled mitochondria in purified cultured RGCs (Fig. 10A) demonstrated an increase in mitochondrial size following NAM supplementation (24 h of pretreatment) which was dose dependent (Fig. 10B). Mitochondria occupied a greater proportion of neurite length (Fig. 10B). Live imaging, analyzed as kymographs (Fig. 10C), revealed a greater number of mitochondria and a striking increase in mitochondrial population mobility and velocity across NAM doses over untreated control (Fig. 10D). Total and stationary mitochondria displayed some variability with NAM dose (Fig. 10D). Larger, more mobile mitochondria may be indicative of increased mitochondrial fusion, with implications for increased OXPHOS capacity (as supported by the increased oxygen consumption rate) and maintenance of energy demands across the cell.

\subsection{Nicotinamide reduces retinal ganglion cell metabolic demands}

Since OXPHOS capacity and mitochondrial motility increased following NAM application, we questioned whether RGC electrical activity was altered. Mouse retina were explanted and incubated with NAM for $2 \mathrm{~h}$ before patch clamping (example profiles displayed in Fig. 11A). At low NAM doses (1-10 mM) the action potential (AP) firing rate was slightly increased, with persistent firing at higher current inputs. Higher NAM doses (100-250 mM), in which we demonstrated neuroprotection in explant retina, demonstrated marked reduction in AP firing rates across current inputs (Fig. 11B). The firing rate (I/O relationship AUC), AP amplitude, and AP rise time were significantly reduced at higher NAM doses (Fig. 11C). Resting membrane potential, AP threshold, and rheobase were not affected by NAM suggesting integrity of the cell's capacity to depolarize (Fig. 11D). These data suggest that the higher neuroprotective doses of NAM lower AP firing frequency in RGCs, without compromising their potential to fire, and thus may reduce RGC metabolic demands. Post-hoc morphological analysis of filled cells confirmed that all recorded cells were RGCs (presence of an axon). RGCs demonstrated broadly similar morphologies between conditions but with some variability (Supplementary Fig. 6). No cells were bi-stratified, and no cells had offset dendritic fields. This suggests that the observed changes to AP metrics are not predominantly the result of differing RGC types. RGCs within the group treated with 1 mM NAM had significantly larger dendritic fields and somas compared to untreated RGCs (control) and as such may contain a different proportion of RGC subtypes which could account for the observed difference in AP metrics in this group.

\section{Discussion}

The control of metabolic homeostasis is essential for normal physiology as well as during stress- and disease-related episodes. To assess this in the context of ocular disease, we introduce a novel, small 
A
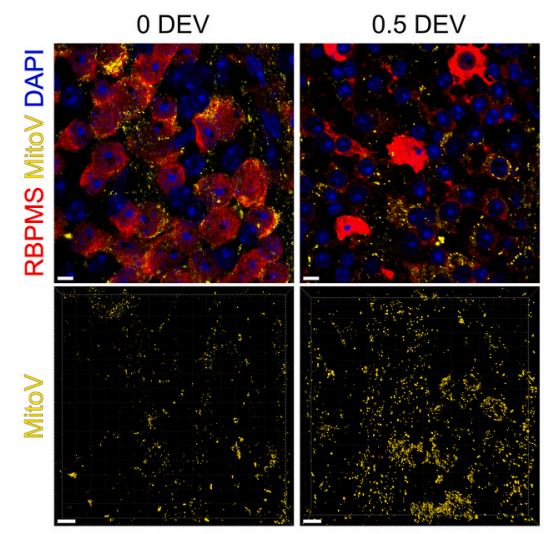

C

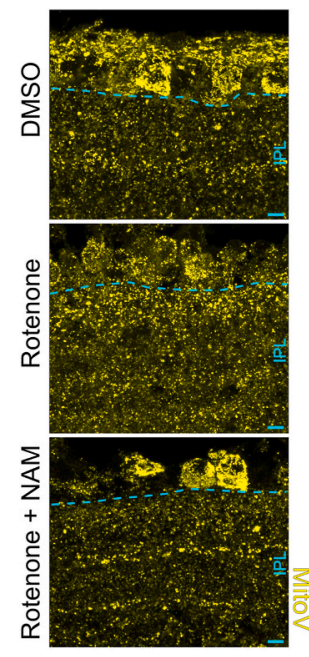

0.5 DEV + NAM

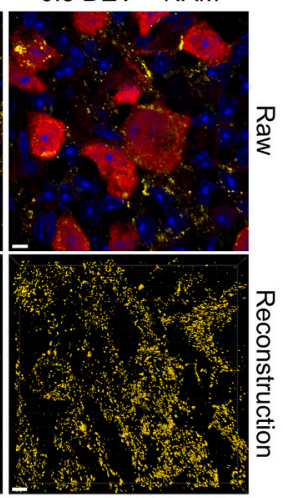

D
B

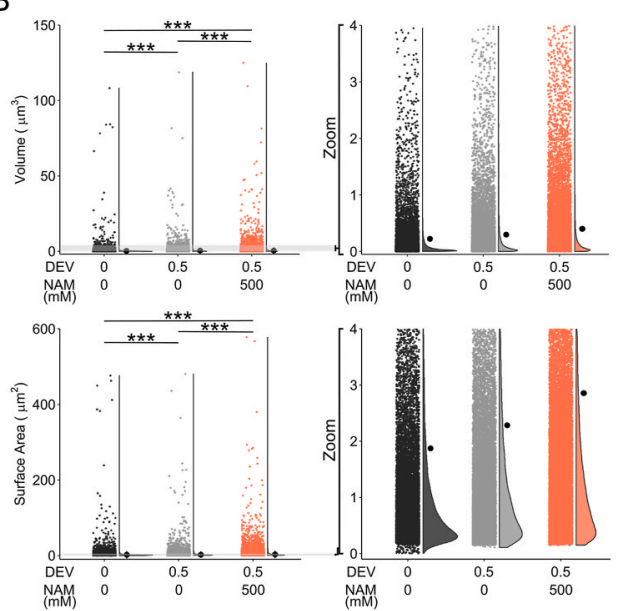

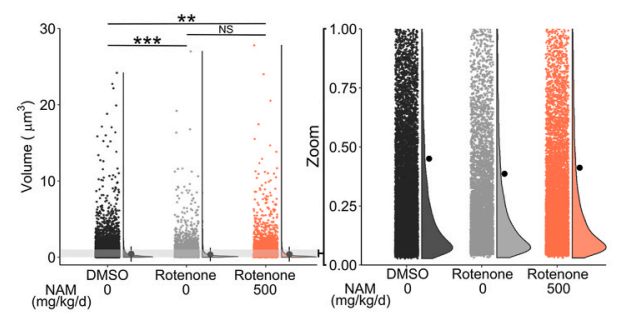

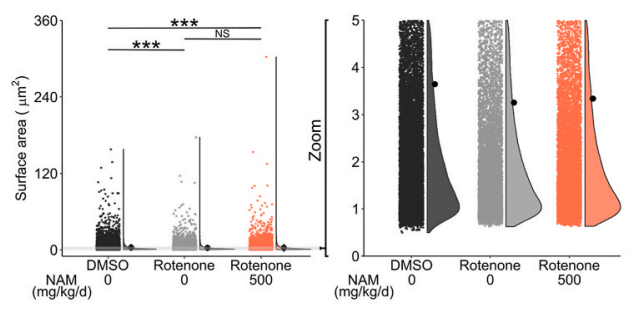

E

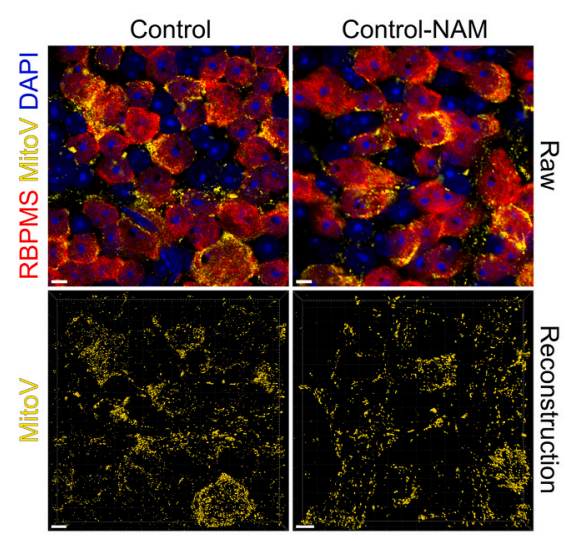

F

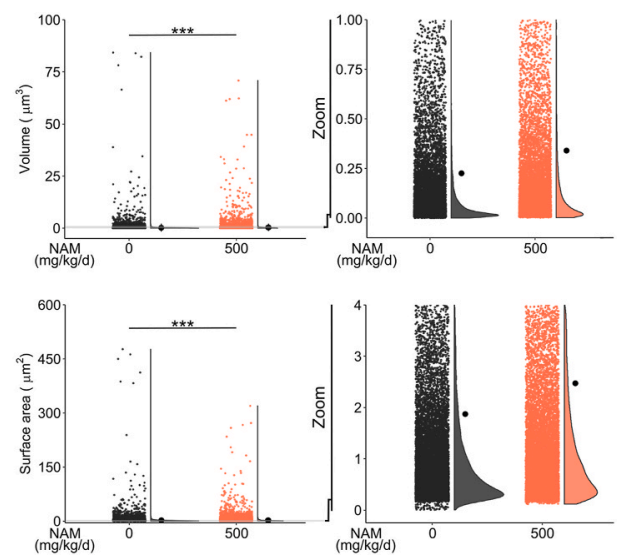

Fig. 9. Nicotinamide increases mitochondrial size. (A) Retinal axotomy culture of MitoV mice (RGC specific YFP labelled mitochondria in the inner retina) maintained for 0.5 days ex vivo (DEV) with or without NAM treated media (and controls fixed at point of euthanasia, 0 DEV) allowed imaging of RGC specific mitochondria in the GFL/RNFL by Airy confocal scanning microscopy following flat mounting ( $n=4$ retina for all conditions). (B) The mean mitochondrial volume and surface area were increased under axotomy, with a further increase in size under NAM treatment $(n=15,216$ disconnected volumes in 0 DEV, 16,765 in 0.5 DEV, 24,558 in $0.5 \mathrm{DEV}+$ NAM). (C) The effects NAM at mitigating direct mitochondrial stress were investigated by intravitreal rotenone injection in MitoV mice $(n=9$ DMSO vehicle treated retina, 3 rotenone, 7 rotenone + NAM $(500 \mathrm{mg} / \mathrm{kg} / \mathrm{d})$ ). Tissue was cryo-sectioned and imaged by Airy confocal scanning microscopy and mitochondria within the IPL (boundary demarcated by cyan broken line). (D) Rotenone caused a reduction in mitochondrial volume relative to controls (DMSO vehicle) in the IPL. NAM had little effect is preventing this change ( $n=15,380$ disconnected volumes in DMSO vehicle treated retinas, 7987 in rotenone treated, 10,418 in rotenone + NAM). These results demonstrate the heterogeneous nature of OHT insult on mitochondria that shares features with both direct RGC and direct mitochondrial stress. (E) In order to determine whether NAM increases mitochondrial size in uninjured retina, MitoV mice were maintained on a NAM diet (500 mg/ $\mathrm{kg} / \mathrm{d}$ ) for 1 week. Retina were fixed immediately after euthanasia and compared to untreated controls by Airy confocal imaging and reconstruction ( $n=4$ control retinas, 4 NAM supplemented). (F) NAM supplementation increased mean mitochondrial volume and surface area in RGCs, supporting observations of mitochondria under stress above ( $n=15,216$ disconnected volumes in control, 13,306 in NAM supplemented). Zoom $=$ inset of data points. Scale bar $=5 \mu \mathrm{m}$ in A, C, and $\mathrm{E}$. $* * P<$ $0.01,{ }^{* * *} P<0.001$. (For interpretation of the references to color in this figure legend, the reader is referred to the Web version of this article.) 
A

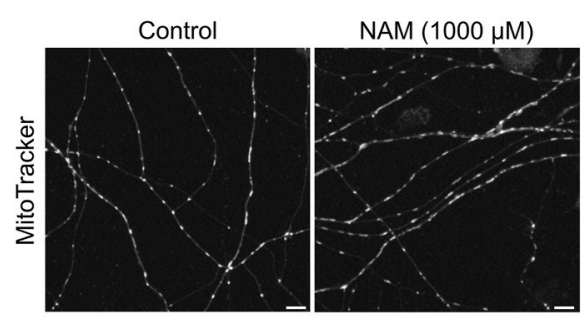

C

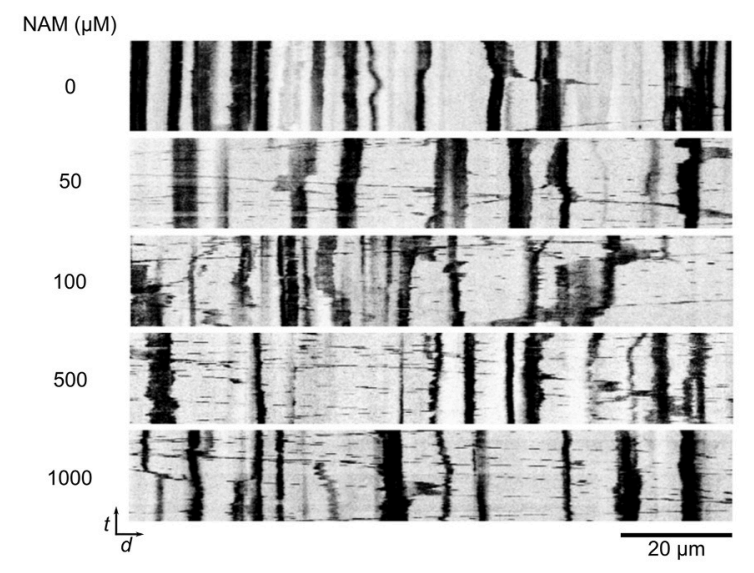

B
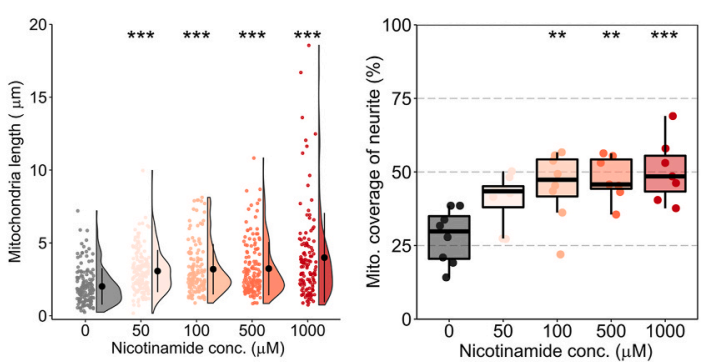

D
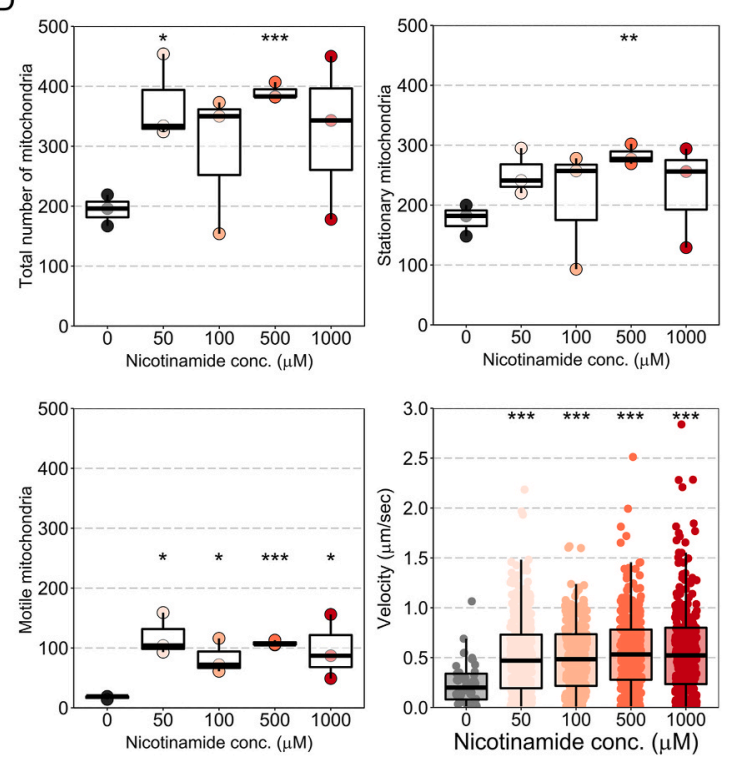

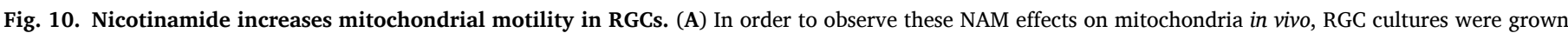

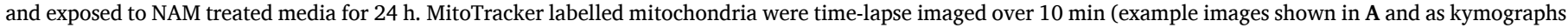

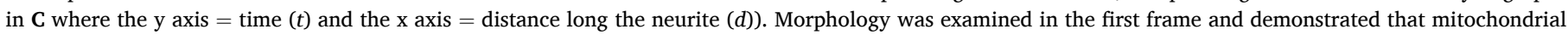

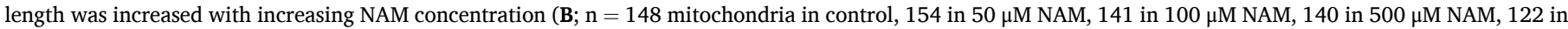

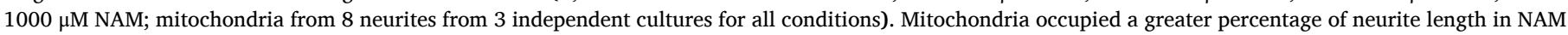

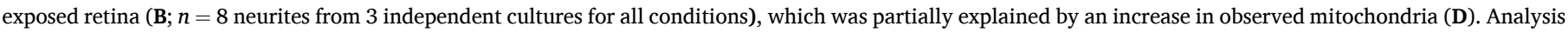

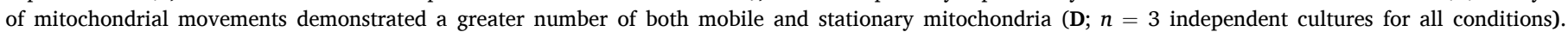

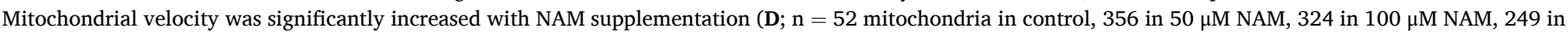

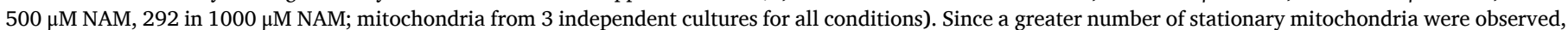

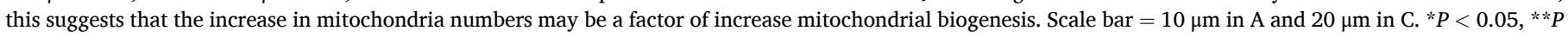
$<0.01, * * * P<0.001$.

molecular weight metabolome for the retina, optic nerve, and superior colliculus of the Brown Norway rat (Rattus norwegicus; the genetic standard for rat). We demonstrate that ocular hypertension causes early metabolic dysregulation that is largely prevented by nicotinamide treatment. These data advance the understanding of nicotinamide as a treatment for glaucoma by demonstrating substantial metabolic protection and robust mitigation of glaucoma-related insults across multiple retinal ganglion cell compartments. Elevated IOP is a major risk factor for glaucoma and is the most significant factor in many patients. We modelled glaucoma in this context with an acute, inducible IOP increase (ocular hypertension) and demonstrate that retinal and optic nerve NAD decline following ocular hypertension in the absence of an age-related effect on NAD homeostasis. It is important to note that significant retinal ganglion cell death has occurred at this time point (which may influence whole tissue NAD metabolism) and that at an earlier 3 day time point (high IOP, but no detectable retinal ganglion cell death [11]) $\mathrm{NAD}$ is yet to decline. In order to better understand the temporal nature of NAD decline and its role in glaucoma pathophysiology it will be important to investigate further disease time points to identify whether ocular hypertension related NAD decline precedes retinal ganglion cell death. Metabolomics revealed a number of significantly altered metabolites in the retina and superior colliculus, but with no detectable changes in the optic nerve at this early 3 day time point. Ocular hypertension does not directly insult the superior colliculus. Therefore, any metabolic disruption to the superior colliculus must arise from retinal ganglion cell mediated processes, not limited to metabolic stress, but could include stresses such as loss of axoplasmic transport [11,34]. We cannot discount early neurodegenerative changes in the optic nerve on the basis of the absence of metabolic dysfunction (as detected using this metabolomics panel). However, the nicotinamide induced metabolic changes observed in normal optic nerve were lost in nicotinamide treated ocular hypertension optic nerves, which may imply subtle optic nerve metabolic changes during ocular hypertension. Crucially, NAD, $\mathrm{NADH}$, and nicotinamide were all significantly elevated in ocular hypertensive nicotinamide treated optic nerves. As NAD is crucial for axon protection [33], this suggests the potential for long term neuroprotection of the optic nerve in glaucoma.

Our previous experiments using the DBA/2J mouse demonstrated that increasing NAD via Nmnat1 gene therapy provides a robust neuroprotection. Over-expression of Nmnat1 provides this neuroprotection outside of the context of increased nicotinamide, but is additive when additional nicotinamide is added to the system [5]. It is therefore reasonable to hypothesize that directly increasing NAD provides retinal ganglion cell neuroprotection in this context, which is supported by the 
A

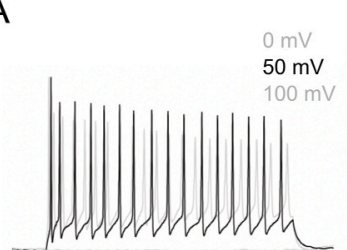

Control (0 mM NAM)

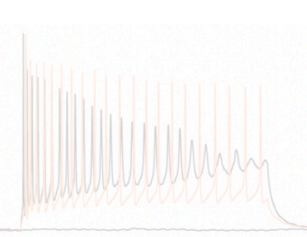

1 mM NAM

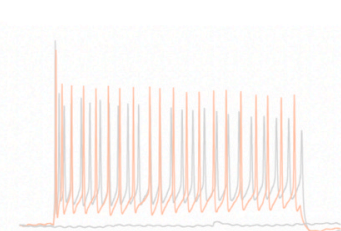

10 mM NAM

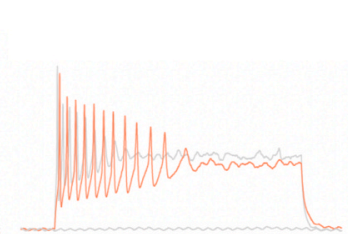

100 mM NAM

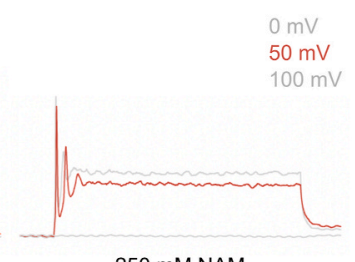

250 mM NAM

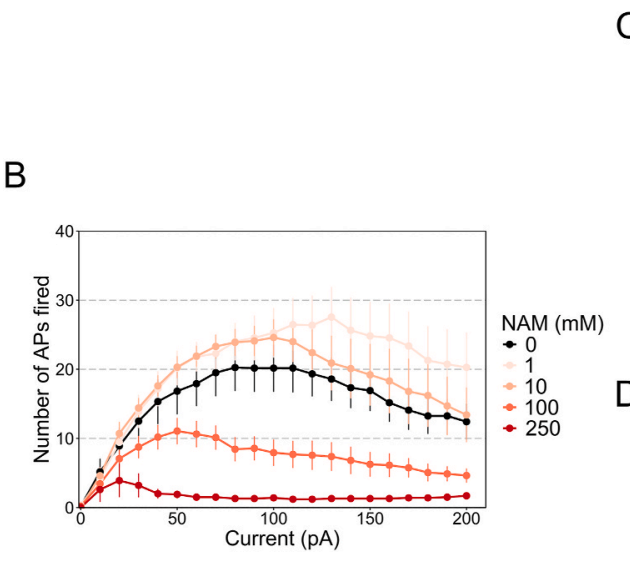

C
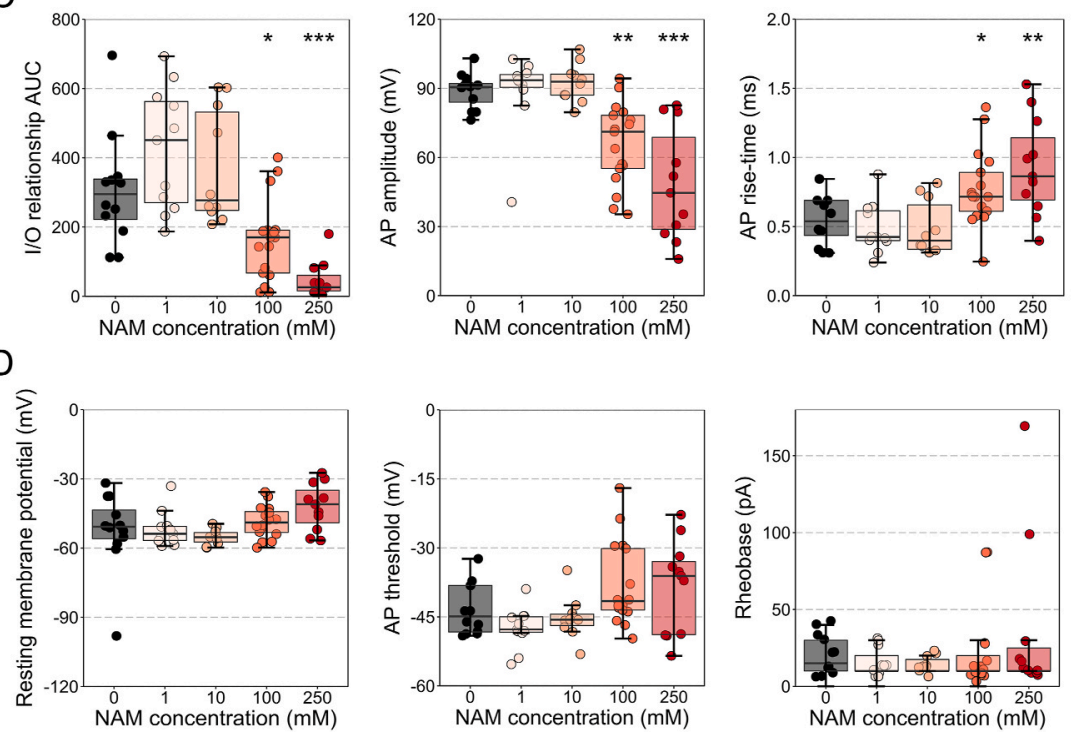

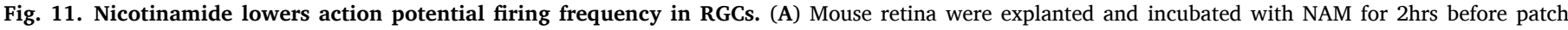

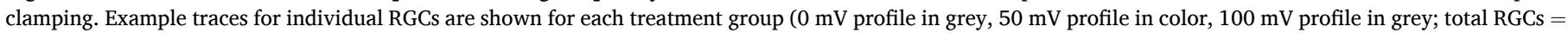

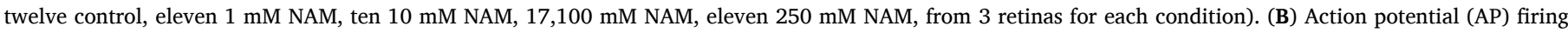

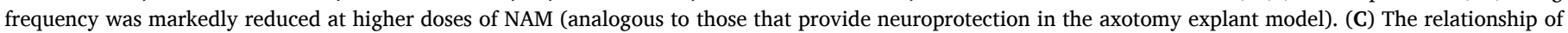

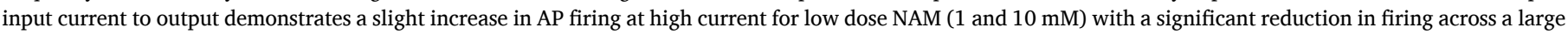

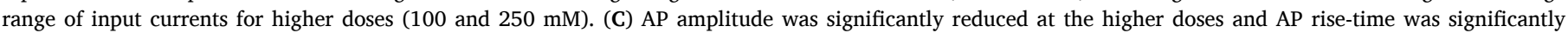

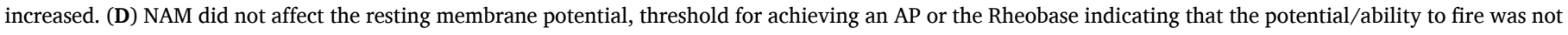

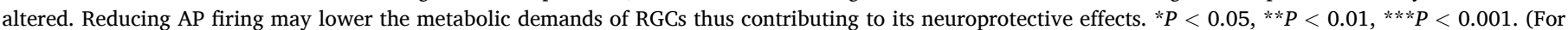
interpretation of the references to color in this figure legend, the reader is referred to the Web version of this article.)

role of NAD in Wallerian degeneration [35]. However, there are other potential metabolic processes afforded by increasing nicotinamide levels that may confer, or partially confer, neuroprotection in addition to nicotinamide's role in elevating NAD. In the nicotinamide treated tissue we cannot differentiate whether these metabolic changes are a consequence of neuroprotection itself or are directly providing neuroprotection. Following ocular hypertension, we observed a number of changed metabolites that may influence retinal ganglion cell neurodegeneration and neuroprotection. Creatine and creatinine were altered in the retina following ocular hypertension. Supporting this, creatine kinase (responsible for the conversion of creatine to phosphocreatine) expression is altered in retinal ganglion cells in the DBA/2J mouse model of glaucoma in response to elevated IOP. $C k b$ (creatine kinase, brain; coupled to glycolysis) is up-regulated and Ckmt1 (creatine kinase, mitochondrial 1; coupled to OXPHOS) is down-regulated [5]. The creatine/phosphocreatine/creatine kinase system provides an important ATP buffer and energy shuttle system within tissues with high energy demand and fluctuation (since phosphocreatine cycling back to creatine generates ATP) [36]. Creatine supplementation is neuroprotective to retinal ganglion cells in cell culture but not in other in vivo in models of retinal excitotoxicity or acute ischemic injury [37]. We observed an increase in $\alpha$-ketoglutaric acid, a key TCA cycle intermediate. $\alpha$-ketoglutarate dehydrogenase catalyzes the conversion of $\alpha$-ketoglutarate to succinyl CoA (an NAD dependent reaction [38]). Reduction in the activity of $\alpha$-ketoglutarate dehydrogenase is well-established in many neurodegenerative diseases with a metabolic component [39]. We observed increased homocysteine in the retina following ocular hypertension, which suggests potential neurotoxic metabolic changes in addition to energy disruption. Increased homocysteine has been described as a marker of a number of neurodegenerative diseases [40] and has been demonstrated to induce retinal ganglion cell death in vitro and in vivo (likely through an excitotoxicity mechanism) [41,42]. Further to this, glycerophosphocholine was also increased in ocular hypertension. Increased glycerophosphocholine is established as an indicator of membrane integrity disruption in neurodegenerative disease [43] created by the breakdown of phosphatidylcholine by phospholipase $A_{2}$. Phospholipase $A_{2}$ inhibitors or genetic knockout of phospholipase $A_{2}$ reduce ischemic neurodegeneration and attenuates Alzheimer's disease in mouse models $[44,45]$. Supporting this, phospholipase $A_{2}$ activation has also been demonstrated in Alzheimer's disease patients and post-mortem tissue $[46,47]$. Taken together, the neuroprotective role of nicotinamide may not be solely limited to its capacity to generate NAD, however these additional metabolites need to be definitively mechanistically tested.

Nicotinamide treatment robustly protected against retinal ganglion cell damaging events. The maintenance of cellular NAD pools is critical for neuronal survival as it provides co-enzyme resources necessary for maintaining a variety of complex cellular functions including ATP production, protein deacetylase activity, calcium homeostasis, conversion to NADPH, and the maintenance of gene expression and DNA repair [33, 
48]. The presence of metabolic dysregulation and ATP depletion $[5,49]$, calcium dysregulation [50], mitochondrial degeneration [4,5], and reactive oxygen species [51] all contribute to glaucoma pathogenesis, supporting a role for NAD as a critical regulator of neuronal health in glaucoma. Nicotinamide supplementation is, therefore, likely to confer multiple neuroprotective effects. These features are not unique to glaucoma and are common to other neurodegenerative diseases in which mitochondrial degeneration and metabolic dysfunction occur. Increasing NAD through multiple substrate avenues has protective effects in many of these diseases, including Alzheimer's disease [52-55], Parkinson's disease [56-59], other neurodegenerations [60,61], and other mitochondrial degenerative disorders [62,63]. Continued elucidation of the mechanisms of Wallerian degeneration has further demonstrated the role of NAD in axon survival. NAD decreases in damaged axons [64], while expression of the $W l d^{S}$ allele or viral overexpression/modified localization of NMNATs are protective, restoring NAD levels [5,65-68]. Increased NAD appears to exhibit numerous functional local roles in this context, including providing sufficient substrate to mitigate local SARM1-dependent NAD consumption and protecting against nicotinamide mononucleotide (NMN) induced axon damage [69,70].

Metabolomic analysis confirmed that retina, optic nerve, and superior colliculus all demonstrated increased NAD following nicotinamide administration. Importantly, nicotinamide drove an increase in NAD in mature in vivo systems and in isolated neurons demonstrating that retinal neurons themselves are capable of utilizing nicotinamide without a requirement for first-pass metabolism or other structural chemical changes. Crucially, high dose nicotinamide caused minor changes to the normal metabolome, mostly influencing metabolites linked directly to NAD or increasing amino acids L-threonine, L-glutamate, L-aspartate, and phenylalanine with limited predicted pathway effects (i.e. low predicted impact on critical pathway nodes via KEGG analysis), suggesting that chronic nicotinamide treatment is unlikely to have substantial deleterious effects in visual system tissue metabolism in the long-term. This is important if nicotinamide is to be considered as a prophylactic in glaucoma patients where significant neurodegeneration is yet to occur. Hierarchical clustering revealed that tissues could be grouped based on the presence or absence of nicotinamide treatment over disease related changes. However, this was largely driven by large fold changes in a small number of metabolites in nicotinamide treatment as opposed to smaller fold changes in numerous metabolites in ocular hypertension. Metabolic stabilization and neuroprotection were achieved with relatively short-term administration of nicotinamide (1 week prophylactic), and dissociated neurons were capable of mobilizing nicotinamide to NAD rapidly (within hours), demonstrating the short period required to achieve an increase in cellular NAD pools. These results support nicotinamide supplementation as a therapeutic strategy for neuroprotection in glaucoma with particular regard to its rapid mobilization to NAD in visual system tissues. While the rodent and human visual system share many common features, and NAD synthesis pathways are conserved, the metabolic consequences in humans could vary. As we focused on small molecular weight metabolites, the consequences to the whole metabolome remain unknown and further metabolomics and lipidomic studies in humans will be necessary especially within the context of clinical trials and long-term testing. To our knowledge, this is the first small molecular weight metabolome for these tissues which will provide a resource and may aid others in the identification of key metabolic changes related to visual pathologies.

The extent to which the NAD increases were restricted to retinal ganglion cells is not known since the metabolomics data is derived from a mixed cell population which included retinal ganglion cell compartments. The optic nerve, containing retinal ganglion cell axons, represents the most retinal ganglion cell enriched tissue based on the relative abundance of cell types, suggesting that retinal ganglion cells likely exhibit increased NAD following nicotinamide supplementation. Increasing NAD across multiple cell types, including glia which offer metabolic support to retinal ganglion cells, may enhance the neuroprotective effect, yet this remains to be explored and is an exciting avenue for therapeutic research, especially in other neurodegenerative diseases in which compromised metabolic function is coupled with a neuroinflammatory components such as Alzheimer's disease. Increasing NAD production by Nmnat1 gene therapy in retinal ganglion cells is neuroprotective and supports a hypothesis in which retinal ganglion cell specific strategies to increase NAD are neuroprotective [5]. Since the intracellular location of NAD is key to its neuroprotective effects [71] further studies examining this in retinal ganglion cells will aid in elucidating the mechanisms of neuroprotection.

We identify mitochondrial protection and oxidative phosphorylation maintenance as potential routes to buffering retinal ganglion cells bioenergetic insufficiency [72-74]. Nicotinamide treatment increased mitochondrial size in normal and disease conditions (ocular hypertension and axotomy, but not rotenone which specifically insults mitochondria). However, differing mitochondrial populations may reflect the health of the neuron rather than a direct nicotinamide effect on mitochondrial morphology. In cultured retinal ganglion cells, nicotinamide also increased basal mitochondrial size. Basal mitochondrial mobility was also significantly increased by nicotinamide. Typically, mitochondrial biogenesis occurs at the soma and anterograde axonal transport moves these new mitochondria down the axon, whereas retrograde axonal transport returns dysfunctional mitochondria to the soma for degradation [75]. Mitochondrial transport is ATP dependent and our data suggest a greater free ATP pool following nicotinamide administration. NAD levels are known to play an important regulatory role in mitochondrial biogenesis and degradation (in particular through SIRT1 and SIRT3 activity, which is increased by NAD [76-78]). Supporting this, DBA/2J mice administered nicotinamide demonstrate an increase in Ppargc1 expression in retinal ganglion cells (encodes PGC-1 $\alpha$; a master regulator of mitochondrial biogenesis) [5].

Electrophysiological analysis of retinal ganglion cells demonstrated that nicotinamide treatment lowered action potential firing rates at the doses associated with neuroprotection. Further experiments will be necessary to corroborate these findings since we only sampled small numbers of retinal ganglion cells. Nicotinamide may elicit differing responses among the different retinal ganglion cell subtypes and using subtype reporter strains would allow isolation of specific subtypes of retinal ganglion cells. Alternatively, the use of multi-electrode arrays would facilitate the capture of hundreds of retinal ganglion cell responses which would deliver sufficient numbers so as to produce populations with similar subtype compositions between comparisons. By washing in/out nicotinamide, the same cell could be followed over time, which would inform on the longevity and/or reversibility of the firing suppression. It should be noted that all of these methods are caveated as the preparation requires an axotomized retina. However, in other tissue preparations exogenous nicotinamide and NAD have demonstrated similar suppression and prolongation of action potential firing and activity (neuronal and cardiac preparations) [79-83]. It is important to note that nicotinamide has been used therapeutically at high doses long-term in clinical preparations without noted incidences of visual or cognitive disturbances $[9,74]$. Pattern electroretinogram (ERG) is a retinal ganglion cell specific functional test, representing a global summation of retinal ganglion cell signals. Nicotinamide supplementation was sufficient to preserve pattern ERG under chronic glaucomatous pathology $[5,84]$ and has demonstrated a recovery in visual function as assessed by ERG in human glaucoma patients [7]. These findings suggest that even if the changes in retinal ganglion cell activity identified by patch clamping were recapitulated in vivo, visual function would not be disturbed. A concomitant decrease in retinal ganglion cell metabolic demand through reduced action potential firing frequency could mitigate the effects of a retinal ganglion cell energy crisis. A reduced energetic load on retinal ganglion cells may partially explain enhanced survival.

These findings advocate furthering clinical trials for the use of 
nicotinamide as a treatment for glaucoma. Nicotinamide has a long established clinical safety profile, with limited long-term side effects. Long-term clinical trials that include individuals with early glaucoma (in which cell loss is heterogeneous and a significant portion of healthy cells likely remain) will be essential in determining the potential for nicotinamide as a prophylactic treatment for glaucoma. However, longerterm treatment of nicotinamide in animals retain their importance, especially in non-human primates which closely recapitulate the human visual system. A number of disease preventative measures have been identified in animal models of glaucoma, which provide robust treatment at early and mid-disease time points, but long-term neuroprotective effects are not maintained or are yet to be demonstrated [18, 85-88]. Animals were treated with high doses of nicotinamide that represent achievable scaled doses to humans (the lowest neuroprotective dose here of $200 \mathrm{mg} / \mathrm{kg} / \mathrm{d}$ in the rat representing $1.9 \mathrm{~g} / \mathrm{day}$ in a $60 \mathrm{~kg}$ human [31]). While we did not test any extra-visual system effects of these doses, the safety of the doses suggested here, as well as doses up to $12 \mathrm{~g} / \mathrm{d}$, is already established [9]. Although some patients may find adherence to these doses difficult to achieve because of the necessary number of tablets (especially when taken in addition to other common medications typical of more elderly patients) and the regularity of dosing, doses in the $1-3 \mathrm{~g} / \mathrm{d}$ range are likely to be suitable for many patients. Supporting this, $3 \mathrm{~g} / \mathrm{d}(6 \times 500 \mathrm{mg}$ capsules $)$ had a high tolerance and compliance in Hui et al. nicotinamide glaucoma clinical trial (94\% adherence).

\section{Conclusions}

Collectively, our data suggest that nicotinamide given as an early intervention or as a prophylactic treatment produces minimal shortterm changes to healthy, non-disease visual system tissue with no observed deleterious effects to normal retinal ganglion cells. Nicotinamide buffers against metabolic and bioenergetic insufficiency to provide a potent neuroprotection against a variety of glaucoma-related stresses. The doses used here are realistic for human treatment regimens and support further clinical trials for glaucoma as well as potentially a range of metabolic and ophthalmic diseases.

\section{Ethics approval}

All breeding and experimental procedures were undertaken in accordance with the Association for Research for Vision and Ophthalmology Statement for the Use of Animals in Ophthalmic and Research. Individual study protocols were approved by Stockholm's Committee for Ethical Animal Research (10,389-2018).

\section{Consent for publication}

Not applicable.

\section{Availability of data and materials}

All data generated or analyzed during this study are included in this published article [and its supplementary information files].

\section{Funding}

Vetenskapsrådet 2018-02124, StratNeuro StartUp grant, Glaucoma Research Foundation Shaffer Grant, Ögonfonden, Stiftelsen Lars Hiertas Minne, Stiftelsen Kronprinsessan Margaretas Arbetsnämnd för synskadade, and Karolinska Institutet Foundation Grants (PAW). Pete Williams is supported by the Karolinska Institutet in the form of a Board of Research Faculty Funded Career Position and by St. Erik Eye Hospital philanthropic donations (PAW). China Scholarship Council 201706100202 (SS). Australian Government Research Training Program Scholarship (SAE). Novo Nordisk Foundation NNF18SA0034956
(RV). Vetenskapsrådet 2019-06076, the Swedish Society for Medical Research, Knut and Alice Wallenberg Foundation, Swedish Research Council, Cronqvist Foundation, and Ögonfonden (GJ). RWB is supported by the National Institutes of Health R37 NS054154, and the generation of the MitoV mice was supported by an ALS Association (RWB). AFA försäkringar, Karolinska Institutet Board of Research senior position support (RB). Velux Foundation 1179261001/2, Fight for Sight Denmark (MK). Fight for Sight UK Studentships 515,905 and 512,264 (JEM). Joan Miller Foundation and Craig and Connie Kimberley Fund (JGC). Marcela Votruba is supported by the School of Vision Sciences, Cardiff University (MV).

\section{Author's contributions}

JRT - designed and performed experiments, analyzed data, wrote the manuscript; AO - performed experiments, analyzed data; SS - performed experiments, analyzed data; SAE - performed experiments, analyzed data; GC - performed experiments, analyzed data; RV - performed experiments, analyzed data; $\mathrm{MJ}$ - performed experiments, analyzed data; EL - performed experiments; APV - analyzed data; AD-V - analyzed data; EK - performed experiments; SR - analyzed data; GJ - provided resources and expertise, designed experiments; PGF - provided resources and expertise, designed experiments; RWB - provided resources and expertise, designed experiments; $\mathrm{RB}$ - provided resources and expertise, designed experiments; MK - provided resources, designed experiments; JEM - provided resources, designed experiments; JGC provided resources, designed experiments; MV - provided resources, designed experiments; PAW - conceived, designed, performed experiments, analyzed data, wrote the manuscript. All authors read and approved the final manuscript.

\section{Declaration of competing interest}

The authors declare that they have no competing interests.

\section{Acknowledgements}

The Authors would like to thank Monica Aronsson and Diana Rydholm for their assistance with animal husbandry and maintenance, St. Erik Eye Hospital for financial support for research space, clinical histopathology, and animal facilities, the Simon John Lab at The Jackson Laboratory for assistance with breeding and shipping animals, the Knut and Alice Wallenberg Foundation and Karolinska Institutet for supporting the CLICK imaging facility, and the Swedish Metabolomics Centre for assistance developing the small molecular weight metabolomics.

\section{List of abbreviations}

$\begin{array}{ll}\text { ADP } & \text { Adenosine diphosphate } \\ \text { AP } & \text { Action potential } \\ \text { ATP } & \text { Adenosine triphosphate } \\ \text { BN } & \text { Brown Norway } \\ \text { DEV } & \text { Days ex vivo } \\ \text { DMEM } & \text { Dulbecco's modified Eagle's medium } \\ \text { DMSO } & \text { Dimethyl sulfoxide } \\ \text { ECR } & \text { Extracellular acidification rate } \\ \text { ERG } & \text { Electroretinogram } \\ \text { GCL } & \text { Ganglion cell layer } \\ \text { HBSS } & \text { Hank's balanced salt solution } \\ \text { HC } & \text { Hierarchical clustering } \\ \text { IOP } & \text { Intraocular pressure } \\ \text { IPL } & \text { Inner plexiform layer } \\ \text { KEGG } & \text { Kyoto Encyclopedia of Genes and Genomes } \\ \text { NAD } & \text { Nicotinamide adenine dinucleotide } \\ \text { NADH } & \text { Nicotinamide adenine dinucleotide }+ \text { hydrogen (reduced) }\end{array}$




$\begin{array}{ll}\text { NAM } & \text { Nicotinamide } \\ \text { NT } & \text { Normotensive } \\ \text { OCR } & \text { Oxygen consumption rate } \\ \text { OCT } & \text { Optical coherence tomography } \\ \text { OHT } & \text { Ocular hypertension } \\ \text { OXPHOS } & \text { Oxidative phosphorylation } \\ \text { PC1 } & \text { Principle component 1 } \\ \text { PC2 } & \text { Principle component } 2 \\ \text { PCA } & \text { Principle component analysis } \\ \text { RBPMS } & \text { RNA-binding protein with multiple splicing } \\ \text { REDOX } & \text { Reduction-oxidation } \\ \text { RGC } & \text { Retinal ganglion cell } \\ \text { RNFL } & \text { Retinal nerve fiber layer } \\ \text { SC } & \text { Superior colliculus } \\ \text { TCA } & \text { Tricarboxylic acid cycle (also citric acid cycle/Krebs cycle) }\end{array}$

\section{Appendix A. Supplementary data}

Supplementary data to this article can be found online at https://doi. org/10.1016/j.redox.2021.101988.

\section{References}

[1] Y.C. Tham, X. Li, T.Y. Wong, H.A. Quigley, T. Aung, C.Y. Cheng, Global prevalence of glaucoma and projections of glaucoma burden through 2040: a systematic review and meta-analysis, Ophthalmology 121 (11) (2014) 2081-2090.

[2] S. Naik, A. Pandey, S.A. Lewis, B.S.S. Rao, S. Mutalik, Neuroprotection: a versatile approach to combat glaucoma, Eur. J. Pharmacol. 881 (2020) 173208.

[3] D. Peters, B. Bengtsson, A. Heijl, Factors associated with lifetime risk of open-angle glaucoma blindness, Acta Ophthalmol. 92 (5) (2014) 421-425.

[4] J.R. Tribble, A. Vasalauskaite, T. Redmond, R.D. Young, S. Hassan, M.P. Fautsch, et al., Midget retinal ganglion cell dendritic and mitochondrial degeneration is an early feature of human glaucoma, Brain Commun 1 (1) (2019) fcz035.

[5] P.A. Williams, J.M. Harder, N.E. Foxworth, K.E. Cochran, V.M. Philip, V. Porciatti, et al., Vitamin B-3 modulates mitochondrial vulnerability and prevents glaucoma in aged mice, Science 355 (6326) (2017) 756-760.

[6] J. Kouassi Nzoughet, J.M. Chao de la Barca, K. Guehlouz, S. Leruez, L. Coulbault, S. Allouche, et al., Nicotinamide deficiency in primary open-angle glaucoma, Invest. Ophthalmol. Vis. Sci. 60 (7) (2019) 2509-2514.

[7] F. Hui, J. Tang, P.A. Williams, M.B. McGuinness, X. Hadoux, R.J. Casson, et al., Improvement in inner retinal function in glaucoma with nicotinamide (vitamin B3) supplementation: a crossover randomized clinical trial, Clin. Exp. Ophthalmol. 48 (7) (2020) 903-914.

[8] B. Poljsak, I. Milisav, Vitamin B3 forms as precursors to NAD+: are they safe? Trends Food Sci. Technol. 79 (2018) 198-203.

[9] M. Knip, I.F. Douek, W.P. Moore, H.A. Gillmor, A.E. McLean, P.J. Bingley, et al., Safety of high-dose nicotinamide: a review, Diabetologia 43 (11) (2000) 1337-1345.

[10] J.E. Morgan, Retina ganglion cell degeneration in glaucoma: an opportunity missed? A review, Clin. Exp. Ophthalmol. 40 (4) (2012) 364-368.

[11] J.R. Tribble, A. Otmani, E. Kokkali, E. Lardner, J.E. Morgan, P.A. Williams, Retinal ganglion cell degeneration in a rat magnetic bead model of ocular hypertensive glaucoma, Transl Vis Sci Technol 10 (1) (2021) 21.

[12] N.D. Bull, T.V. Johnson, G. Welsapar, N.W. DeKorver, S.I. Tomarev, K.R. Martin, Use of an adult rat retinal explant model for screening of potential retinal ganglion cell neuroprotective therapies, Invest. Ophthalmol. Vis. Sci. 52 (6) (2011) 3309-3320.

[13] K.E. Binley, W.S. Ng, Y.A. Barde, B. Song, J.E. Morgan, Brain-derived neurotrophic factor prevents dendritic retraction of adult mouse retinal ganglion cells, Eur. J. Neurosci. 44 (3) (2016) 2028-2039.

[14] X. Zhang, D. Jones, F. Gonzalez-Lima, Neurodegeneration produced by rotenone in the mouse retina: a potential model to investigate environmental pesticide contributions to neurodegenerative diseases, J. Toxicol. Environ. Health 69 (18) (2006) 1681-1697.

[15] K. Cimdins, H.S. Waugh, V. Chrysostomou, M.I.G. Lopez Sanchez, V.A. Johannsen, M.J. Cook, et al., Amyloid precursor protein mediates neuronal protection from rotenone toxicity, Mol. Neurobiol. 56 (8) (2019) 5471-5482.

[16] J. Schindelin, I. Arganda-Carreras, E. Frise, V. Kaynig, M. Longair, T. Pietzsch, et al., Fiji: an open-source platform for biological-image analysis, Nat. Methods 9 (7) (2012) 676-682.

[17] L. Wang, J. Dong, G. Cull, B. Fortune, G.A. Cioffi, Varicosities of intraretinal ganglion cell axons in human and nonhuman primates, Invest. Ophthalmol. Vis. Sci. 44 (1) (2003) 2-9.

[18] P.A. Williams, J.R. Tribble, K.W. Pepper, S.D. Cross, B.P. Morgan, J.E. Morgan, et al., Inhibition of the classical pathway of the complement cascade prevents early dendritic and synaptic degeneration in glaucoma, Mol. Neurodegener. 11 (2016).

[19] P.M. Quiros, A. Goyal, P. Jha, J. Auwerx, Analysis of mtDNA/nDNA ratio in mice, Curr Protoc Mouse Biol 7 (1) (2017) 47-54.
[20] J. Xia, N. Psychogios, N. Young, D.S. Wishart, MetaboAnalyst: a web server for metabolomic data analysis and interpretation, Nucleic Acids Res. 37 (2009) W652-W660. Web Server issue.

[21] J. Xia, D.S. Wishart, Web-based inference of biological patterns, functions and pathways from metabolomic data using MetaboAnalyst, Nat. Protoc. 6 (6) (2011) 743-760.

[22] R.A. van den Berg, H.C. Hoefsloot, J.A. Westerhuis, A.K. Smilde, M.J. van der Werf, Centering, scaling, and transformations: improving the biological information content of metabolomics data, BMC Genom. 7 (2006) 142

[23] Z. Gu, L. Gu, R. Eils, M. Schlesner, B. Brors, Circlize Implements and enhances circular visualization in R, Bioinformatics 30 (19) (2014) 2811-2812.

[24] T. Misgeld, M. Kerschensteiner, F.M. Bareyre, R.W. Burgess, J.W. Lichtman, Imaging axonal transport of mitochondria in vivo, Nat. Methods 4 (7) (2007) $559-561$.

[25] R.W. Burgess, P.G. Fuerst, Distinct expression patterns of mitochondrially localized YFP in neuronal subsets in the retina of three transgenic mouse lines, BMC Res. Notes 3 (2010) 253.

[26] S. Sun, I. Erchova, F. Sengpiel, M. Votruba, Opa1 deficiency leads to diminished mitochondrial bioenergetics with compensatory increased mitochondrial motility, Invest. Ophthalmol. Vis. Sci. 61 (6) (2020) 42.

[27] A. Winzeler, J.T. Wang, Purification and culture of retinal ganglion cells from rodents, Cold Spring Harb. Protoc. 2013 (7) (2013) 643-652.

[28] S. Neumann, R. Chassefeyre, G.E. Campbell, S.E. Encalada, KymoAnalyzer: a software tool for the quantitative analysis of intracellular transport in neurons, Traffic 18 (1) (2017) 71-88.

[29] D.M. Skytt, A.K. Toft-Kehler, C.T. Brændstrup, S. Cejvanovic, I.S. Gurubaran, L. $\mathrm{H}$. Bergersen, et al., Glia-neuron interactions in the retina can Be studied in cocultures of müller cells and retinal ganglion cells, BioMed Res. Int. 2016 (2016) 1087647.

[30] R. Vohra, B.I. Aldana, G. Bulli, D.M. Skytt, H. Waagepetersen, L.H. Bergersen, et al., Lactate-mediated protection of retinal ganglion cells, J. Mol. Biol. 431 (9) (2019) 1878-1888.

[31] A.B. Nair, S. Jacob, A simple practice guide for dose conversion between animals and human, J. Basic Clin. Pharm. 7 (2) (2016) 27-31.

[32] K. Kasischke, M. Büchner, A.C. Ludolph, M.W. Riepe, Nuclear shrinkage in live mouse hippocampal slices, Acta Neuropathol. 101 (5) (2001) 483-490.

[33] E. Verdin, $\mathrm{NAD}^{+}$in aging, metabolism, and neurodegeneration, Science 350 (6265) (2015) 1208-1213.

[34] J.R. Tribble, E. Kokkali, A. Otmani, F. Plastino, E. Lardner, R. Vohra, et al., When is a control not a control? Reactive microglia occur throughout the control contralateral pathway of retinal ganglion cell projections in experimental glaucoma, Transl Vis Sci Technol 10 (1) (2021) 22.

[35] M.P. Coleman, A. Höke, Programmed axon degeneration: from mouse to mechanism to medicine, Nat. Rev. Neurosci. 21 (4) (2020) 183-196.

[36] E. Béard, O. Braissant, Synthesis and transport of creatine in the CNS: importance for cerebral functions, J. Neurochem. 115 (2) (2010) 297-313.

[37] P.I. Sia, J.P.M. Wood, G. Chidlow, R. Casson, Creatine is neuroprotective to retinal neurons in vitro but not in vivo, Invest. Ophthalmol. Vis. Sci. 60 (13) (2019) 4360-4377.

[38] L. Tretter, V. Adam-Vizi, Alpha-ketoglutarate dehydrogenase: a target and generator of oxidative stress, Philos. Trans. R. Soc. Lond. B Biol. Sci. 360 (1464) (2005) 2335-2345.

[39] G.E. Gibson, L.C. Park, K.F. Sheu, J.P. Blass, N.Y. Calingasan, The alphaketoglutarate dehydrogenase complex in neurodegeneration, Neurochem. Int. 36 (2) (2000) 97-112.

[40] W. Herrmann, R. Obeid, Homocysteine: a biomarker in neurodegenerative diseases, Clin. Chem. Lab. Med. 49 (3) (2011) 435-441.

[41] S. Navneet, J. Zhao, J. Wang, B. Mysona, S. Barwick, N. Ammal Kaidery, et al. Hyperhomocysteinemia-induced death of retinal ganglion cells: the role of Müller glial cells and NRF2, Redox Biol 24 (2019) 101199.

[42] P. Moore, A. El-sherbeny, P. Roon, P.V. Schoenlein, V. Ganapathy, S.B. Smith, Apoptotic cell death in the mouse retinal ganglion cell layer is induced in vivo by the excitatory amino acid homocysteine, Exp. Eye Res. 73 (1) (2001) 45-57.

[43] J. Klein, Membrane breakdown in acute and chronic neurodegeneration: focus on choline-containing phospholipids, J. Neural. Transm. 107 (8-9) (2000) 1027-1063.

[44] J.V. Bonventre, Z. Huang, M.R. Taheri, E. O’Leary, E. Li, M.A. Moskowitz, et al. Reduced fertility and postischaemic brain injury in mice deficient in cytosolic phospholipase A2, Nature 390 (6660) (1997) 622-625.

[45] B. Qu, Y. Gong, J.M. Gill, K. Kenney, R. Diaz-Arrastia, Heterozygous knockout of cytosolic phospholipase A, Brain Res. 1670 (2017) 248-252.

[46] M. Bárány, Y.C. Chang, C. Arús, T. Rustan, W.H. Frey, Increased glycerol-3phosphorylcholine in post-mortem Alzheimer's brain, Lancet 1 (8427) (1985) 517.

[47] A. Walter, U. Korth, M. Hilgert, J. Hartmann, O. Weichel, K. Fassbender, et al., Glycerophosphocholine is elevated in cerebrospinal fluid of Alzheimer patients, Neurobiol. Aging 25 (10) (2004) 1299-1303.

[48] S. Johnson, S.I. Imai, NAD ${ }^{+}$biosynthesis, aging, and disease, F1000Res 7 (2018) 132, https://doi.org/10.12688/f1000research.12120.1.

[49] S. Baltan, D.M. Inman, C.A. Danilov, R.S. Morrison, D.J. Calkins, P.J. Horner, Metabolic vulnerability disposes retinal ganglion cell axons to dysfunction in a model of glaucomatous degeneration, J. Neurosci. 30 (16) (2010) 5644-5652.

[50] S.D. Crish, D.J. Calkins, Neurodegeneration in glaucoma: progression and calciumdependent intracellular mechanisms, Neuroscience 176 (2011) 1-11.

[51] V. Chrysostomou, F. Rezania, I.A. Trounce, J.G. Crowston, Oxidative stress and mitochondrial dysfunction in glaucoma, Curr. Opin. Pharmacol. 13 (1) (2013) $12-15$. 
[52] Y. Hou, S. Lautrup, S. Cordonnier, Y. Wang, D.L. Croteau, E. Zavala, et al., NAD ${ }^{+}$ supplementation normalizes key Alzheimer's features and DNA damage responses in a new AD mouse model with introduced DNA repair deficiency, Proc Natl Acad Sci U S A. 115 (8) (2018) E1876-E1885, https://doi.org/10.1073/ pnas.1718819115.

[53] B. Gong, Y. Pan, P. Vempati, W. Zhao, L. Knable, L. Ho, et al., Nicotinamide riboside restores cognition through an upregulation of proliferator-activated receptor- $\gamma$ coactivator $1 \alpha$ regulated $\beta$-secretase 1 degradation and mitochondrial gene expression in Alzheimer's mouse models, Neurobiol. Aging 34 (6) (2013) 1581-1588.

[54] A.N. Long, K. Owens, A.E. Schlappal, T. Kristian, P.S. Fishman, R.A. Schuh, Effect of nicotinamide mononucleotide on brain mitochondrial respiratory deficits in an Alzheimer's disease-relevant murine model, BMC Neurol. 15 (2015) 19.

[55] V. Sorrentino, M. Romani, L. Mouchiroud, J.S. Beck, H. Zhang, D. D'Amico, et al., Enhancing mitochondrial proteostasis reduces amyloid- $\beta$ proteotoxicity, Nature 552 (7684) (2017) 187-193.

[56] S. Lehmann, S.H. Loh, L.M. Martins, Enhancing NAD ${ }^{+}$salvage metabolism is neuroprotective in a PINK1 model of Parkinson's disease, Biol Open 6 (2) (2017) 141-147, https://doi.org/10.1242/bio.022186.

[57] H. Jia, X. Li, H. Gao, Z. Feng, L. Zhao, X. Jia, et al., High doses of nicotinamide prevent oxidative mitochondrial dysfunction in a cellular model and improve motor deficit in a Drosophila model of Parkinson's disease, J. Neurosci. Res. 86 (9) (2008) 2083-2090.

[58] J.M. Alisky, Niacin improved rigidity and bradykinesia in a Parkinson's disease patient but also caused unacceptable nightmares and skin rash-a case report, Nutr. Neurosci. 8 (5-6) (2005) 327-329.

[59] D.C. Schöndorf, D. Ivanyuk, P. Baden, A. Sanchez-Martinez, S. De Cicco, C. Yu, et al., The NAD + precursor nicotinamide riboside rescues mitochondrial defects and neuronal loss in iPSC and fly models of Parkinson's disease, Cell Rep. 23 (10) (2018) 2976-2988.

[60] J.B. Lin, S. Kubota, N. Ban, M. Yoshida, A. Santeford, A. Sene, et al., NAMPTmediated $\mathrm{NAD}(+)$ biosynthesis is essential for vision in mice, Cell Rep. 17 (1) (2016) 69-85.

[61] H.W. Liu, C.B. Smith, M.S. Schmidt, X.A. Cambronne, M.S. Cohen, M.E. Migaud, et al., Pharmacological bypass of NAD ${ }^{+}$salvage pathway protects neurons from chemotherapy-induced degeneration, Proc. Natl. Acad. Sci. U. S. A. 115 (42) (2018) 10654-10659, https://doi.org/10.1073/pnas.1809392115.

[62] N.A. Khan, M. Auranen, I. Paetau, E. Pirinen, L. Euro, S. Forsström, et al., Effective treatment of mitochondrial myopathy by nicotinamide riboside, a vitamin B3, EMBO Mol. Med. 6 (6) (2014) 721-731.

[63] E. Pirinen, M. Auranen, N.A. Khan, V. Brilhante, N. Urho, A. Pessia, et al., Niacin Cures Systemic NAD ${ }^{+}$Deficiency and Improves Muscle Performance in AdultOnset Mitochondrial Myopathy, Cell Metabol. 31 (6) (2020) 1078-1090, https:// doi.org/10.1016/j.cmet.2020.04.008, e5.

[64] J. Wang, Q. Zhai, Y. Chen, E. Lin, W. Gu, M.W. McBurney, et al., A local mechanism mediates NAD-dependent protection of axon degeneration, J. Cell Biol. 170 (3) (2005) 349-355.

[65] T. Araki, Y. Sasaki, J. Milbrandt, Increased nuclear NAD biosynthesis and SIRT1 activation prevent axonal degeneration, Science 305 (5686) (2004) 1010-1013.

[66] E. Babetto, B. Beirowski, L. Janeckova, R. Brown, J. Gilley, D. Thomson, et al., Targeting NMNAT1 to axons and synapses transforms its neuroprotective potency in vivo, J. Neurosci. 30 (40) (2010) 13291-13304.

[67] Y. Feng, T. Yan, J. Zheng, X. Ge, Y. Mu, Y. Zhang, et al., Overexpression of Wld(S) or Nmnat2 in mauthner cells by single-cell electroporation delays axon degeneration in live zebrafish, J. Neurosci. Res. 88 (15) (2010) 3319-3327.

[68] P.A. Williams, J.M. Harder, N.E. Foxworth, B.H. Cardozo, K.E. Cochran, S.W. M. John, Nicotinamide and WLDS act together to prevent neurodegeneration in glaucoma, Front. Neurosci. 11 (2017).
[69] M. Di Stefano, I. Nascimento-Ferreira, G. Orsomando, V. Mori, J. Gilley, R. Brown, et al., A rise in NAD precursor nicotinamide mononucleotide (NMN) after injury promotes axon degeneration, Cell Death Differ. 22 (5) (2015) 731-742.

[70] J. Gerdts, E.J. Brace, Y. Sasaki, A. DiAntonio, J. Milbrandt, SARM1 activation triggers axon degeneration locally via NAD ${ }^{+}$destruction, Science 348 (6233) (2015) 453-457.

[71] J. Gilley, M.P. Coleman, Endogenous Nmnat2 is an essential survival factor for maintenance of healthy axons, PLoS Biol. 8 (1) (2010), e1000300.

[72] L.E. Fry, E. Fahy, V. Chrysostomou, F. Hui, J. Tang, P. van Wijngaarden, et al., The coma in glaucoma: retinal ganglion cell dysfunction and recovery, Prog. Retin. Eye Res. 65 (2018) 77-92.

[73] R.J. Casson, G. Chidlow, J.G. Crowston, P.A. Williams, J.P.M. Wood, Retinal energy metabolism in health and glaucoma, Prog. Retin. Eye Res. (2020) 100881.

[74] P.A. Williams, J.M. Harder, S.W.M. John, Glaucoma as a metabolic optic neuropathy: making the case for nicotinamide treatment in glaucoma, J. Glaucoma 26 (12) (2017) 1161-1168.

[75] H. Chen, D.C. Chan, Mitochondrial dynamics-fusion, fission, movement, and mitophagy-in neurodegenerative diseases, Hum. Mol. Genet. 18 (R2) (2009) R169-R176.

[76] J. Milner, Cellular regulation of SIRT1, Curr. Pharmaceut. Des. 15 (1) (2009) 39-44.

[77] J. Brenmoehl, A. Hoeflich, Dual control of mitochondrial biogenesis by sirtuin 1 and sirtuin 3, Mitochondrion 13 (6) (2013) 755-761.

[78] A. Benigni, L. Perico, D. Macconi, Mitochondrial dynamics is linked to longevity and protects from end-organ injury: the emerging role of sirtuin 3, Antioxidants Redox Signal. 25 (4) (2016) 185-199.

[79] M. Liang, X.L. Yin, L.Y. Wang, W.H. Yin, N.Y. Song, H.B. Shi, et al., NAD+ attenuates bilirubin-induced hyperexcitation in the ventral cochlear nucleus by inhibiting excitatory neurotransmission and neuronal excitability, Front. Cell. Neurosci. 11 (2017) 21.

[80] J.M. Bold, C.R. Gardner, R.J. Walker, Central effects of nicotinamide and inosine which are not mediated through benzodiazepine receptors, Br. J. Pharmacol. 84 (3) (1985) 689-696.

[81] K.B. Pustovit, D.V. Abramochkin, Effects of nicotinamide adenine dinucleotide $(\mathrm{NAD}(+))$ and diadenosine tetraphosphate (Ap4A) on electrical activity of working and pacemaker atrial myocardium in Guinea pigs, Bull. Exp. Biol. Med. 160 (6) (2016) 733-736.

[82] V.S. Kuzmin, K.B. Pustovit, D.V. Abramochkin, Effects of exogenous nicotinamide adenine dinucleotide $(\mathrm{NAD}+)$ in the rat heart are mediated by P2 purine receptors, J. Biomed. Sci. 23 (1) (2016) 50

[83] K.B. Pustovit, V.M. Potekhina, A.D. Ivanova, A.M. Petrov, D.V. Abramochkin, V. S. Kuzmin, Extracellular ATP and $\beta$-NAD alter electrical properties and cholinergic effects in the rat heart in age-specific manner, Purinergic Signal. 15 (1) (2019) $107-117$.

[84] T.H. Chou, G.L. Romano, R. Amato, V. Porciatti, Nicotinamide-rich diet in DBA/2J mice preserves retinal ganglion cell metabolic function as assessed by PERG adaptation to flicker, Nutrients 12 (7) (2020).

[85] G.R. Howell, I. Soto, X. Zhu, M. Ryan, D.G. Macalinao, G.L. Sousa, et al., Radiation treatment inhibits monocyte entry into the optic nerve head and prevents neuronal damage in a mouse model of glaucoma, J. Clin. Invest. 122 (4) (2012) 1246-1261.

[86] A.J. Weber, C.D. Harman, BDNF preserves the dendritic morphology of alpha and beta ganglion cells in the cat retina after optic nerve injury, Invest. Ophthalmol. Vis. Sci. 49 (6) (2008) 2456-2463.

[87] J. Agostinone, L. Alarcon-Martinez, C. Gamlin, W.Q. Yu, R.O.L. Wong, A. Di Polo, Insulin signalling promotes dendrite and synapse regeneration and restores circuit function after axonal injury, Brain 141 (7) (2018) 1963-1980.

[88] R.H. Foxton, A. Finkelstein, S. Vijay, A. Dahlmann-Noor, P.T. Khaw, J.E. Morgan, et al., VEGF-A is necessary and sufficient for retinal neuroprotection in models of experimental glaucoma, Am. J. Pathol. 182 (4) (2013) 1379-1390. 